\title{
Diagnostic Pressure Equation as a Weak Constraint in a Storm-Scale Three-Dimensional Variational Radar Data Assimilation System
}

\author{
GUOQING Ge \\ Center for Analysis and Prediction of Storms, University of Oklahoma, Norman, Oklahoma \\ JIDONG GAO \\ NOAA/National Severe Storm Laboratory, Norman, Oklahoma \\ MiNG XUE \\ Center for Analysis and Prediction of Storms, and School of Meteorology, University of Oklahoma, \\ Norman, Oklahoma
}

(Manuscript received 16 November 2011, in final form 20 March 2012)

\begin{abstract}
A diagnostic pressure equation is incorporated into a storm-scale three-dimensional variational data assimilation (3DVAR) system in the form of a weak constraint in addition to a mass continuity equation constraint (MCEC). The goal of this diagnostic pressure equation constraint (DPEC) is to couple different model variables to help build a more dynamic consistent analysis, and therefore improve the data assimilation results and subsequent forecasts. Observational System Simulation Experiments (OSSEs) are first performed to examine the impact of the pressure equation constraint on storm-scale radar data assimilation using an idealized tornadic thunderstorm simulation. The impact of MCEC is also investigated relative to that of DPEC. It is shown that DPEC can improve the data assimilation results slightly after a given period of data assimilation. Including both DPEC and MCEC yields the best data assimilation results. Sensitivity tests show that MCEC is not very sensitive to the choice of its weighting coefficients in the cost function, while DPEC is more sensitive and its weight should be carefully chosen. The updated 3DVAR system with DPEC is further applied to the 5 May 2007 Greensburg, Kansas, tornadic supercell storm case assimilating real radar data. It is shown that the use of DPEC can speed up the spinup of precipitation during the intermittent data assimilation process and also improve the follow-on forecast in terms of the general evolution of storm cells and mesocyclone rotation near the time of observed tornado.
\end{abstract}

\section{Introduction}

There are many challenges in forecasting convective storms. One of them is how to produce a dynamic consistent initial condition for storm-scale numerical weather prediction (NWP) (see the appendix for acronym expansions and variable definitions) models. Currently, the Weather Surveillance Radar-1988 Doppler (WSR-88D) network is the only source of routine observations in the United States that can resolve storm-scale features at

Corresponding author address: Dr. Jidong Gao, 120 David L. Boren Blvd., National Severe Storm Laboratory, Norman, OK 73072.

E-mail: jidong.gao@noaa.gov high enough spatial and temporal resolutions. Therefore, in recent years, many studies are focused on the assimilation of these radar data into NWP models to provide better initial conditions (e.g., Crook and Tuttle 1994; Sun and Crook 1997; Sun and Crook 2001; Hu et al. 2006a,b; Stensrud and Gao 2010). The problem is challenging because the radar only observes a few parameters, typically limited to the radial velocity and reflectivity, while most state variables have to be "retrieved" in the data assimilation (DA) process.

Several DA methods have been applied to the radar DA problem; they include three-dimensional variational data assimilation (3DVAR), four-dimensional variational data assimilation (4DVAR), and ensemble Kalman filter (EnKF). The 4DVAR method is attractive because it 
uses an NWP model as a strong constraint and tries to fit the model state to observations taken at different times during the assimilation window, thereby producing a dynamically consistent state and retrieving unobserved variables. Sun and Crook $(1997,1998)$ and Sun (2005) had shown encouraging results with a 4DVAR based on a cloud model. However, the need to develop and maintain complex adjoint codes for NWP models and the difficulties encountered with complex ice microphysics that are important for storm-scale predictions have limited the adoption of the 4DVAR method in storm-scale NWP operations. The EnKF is an emerging technique, which promises to produce similar assimilation quality as 4DVAR, but avoids the coding of an adjoint model. Many radar DA studies have been carried out in recent years with EnKF (e.g., Snyder and Zhang 2003; Zhang et al. 2004; Caya et al. 2005; Tong and Xue 2005; Xue et al. 2006; Aksoy et al. 2009; Zhang et al. 2009; Aksoy et al. 2010; Dowell et al. 2011; Snook et al. 2011). These studies have shown the great potential of the EnKF method. On the other hand, EnKF is not as mature as the variational methods and so far successful applications to real data assimilation problems are still limited. Computationally, it has similar costs to 4DVAR.

The 3DVAR method is computationally much more efficient, making its real-time applications much more practical. Some studies (e.g., Hu et al. 2006a,b; Hu and Xue 2007a; Stensrud and Gao 2010) have successfully demonstrated the ability of the 3DVAR to assimilate radar data to predict tornadic supercell storms. The Advanced Regional Prediction System (ARPS; Xue et al. 2000, 2001, 2003) 3DVAR system and its cloud analysis package have been used to produce continental U.S.-scale real-time weather predictions at up to $1-\mathrm{km}$ resolution (Xue et al. 2008, 2011). However, despite its successful applications, the 3DVAR method is often challenged by its theoretical suboptimality resulting from its use of static background error covariance and the lack of suitable balances among model variables. Research efforts have been made to incorporate flow-dependent background error covariance in a 3DVAR framework. For example, Liu and Xue (2006) and Liu et al. (2007) reported efforts to build flow-dependent background error covariance in a 3DVAR system using anisotropic recursive filters and demonstrated improvement in moisture retrieval from GPS slant-path water vapor observations. Hamill and Snyder (2000), Lorenc (2003), Buehner (2005), and Wang et al. (2008a,b) advocated a hybrid approach that incorporates flow-dependent background covariance derived from an forecast ensemble into a 3DVAR framework, and the method is recently applied to a radar DA problem for a landfalling hurricane
(Li et al. 2012). The similar methodology can be extended to 4DVAR also. Because of the use of an ensemble, the method is also expensive and requires much research.

Another alternative to improve the balance among model variables in the 3DVAR analysis is to include suitable weak constraints in the 3DVAR cost function, which also helps spread observational information to state variables not directly observed. Gao et al. (1999, 2001, 2004), Hu et al. (2006a,b) and Hu and Xue (2007b) incorporated an anelastic mass continuity equation into the cost function of the ARPS 3DVAR system as a weak constraint and found that this constraint can improve the wind analysis and subsequent storm forecasts. However, there is no direct linkage between the wind and thermodynamic (pressure and temperature) variables in the system. Xiao et al. (2005) reported their efforts to build linkages between the wind and thermodynamic fields in the Fifth-Generation NCAR/Penn State Mesoscale Mode) MM5 3DVAR system by using a constraint based on a linearized Richardson equation, which is derived from mass continuity, adiabatic thermodynamic, and hydrostatic equations. The hydrostatic and adiabatic assumptions in their study are not suitable for the storm-scale DA, however. Some other 3DVAR radar analysis and assimilation studies (e.g., Protat and Zawadzki 2000; Liou 2001; Protat et al. 2001; Weygandt et al. 2002a,b; Liou et al. 2003; Zhao et al. 2006, 2008; Liou and Chang 2009) turned to a two-step approach, where a thermodynamic retrieval technique is applied to derive the temperature and pressure fields from several time levels of retrieved wind fields. This technique was pioneered by Gal-Chen (1978) and Hane and Scott (1978). One of the difficulties with such an approach lies with the accurate retrieval of three wind components and their time tendencies, which by itself is a difficult problem. The results are especially sensitive to the estimate of the time tendencies (Crook 1994).

In the equation constraint to be introduced in this study, the calculation of the wind tendency term is avoided by applying the divergence operator to the three model momentum equations. The derived equation is the diagnostic pressure equation in typical anelastic systems where three momentum tendency terms cancel out. This diagnostic pressure equation is incorporated into our 3DVAR cost function in the form of a weak constraint in addition to the aforementioned mass continuity equation constraint (MCEC). The main goal of this constraint is to improve the consistency between dynamic and thermodynamic fields. Xu et al. (2001) tried to include a similar constraint in their simple adjoint system for retrieving three-dimensional winds from single-Doppler radar that treats the radial velocity as a tracer. They found 
that the DPEC can help improve the retrieval of wind fields in single time data analysis. However, the impact of DPEC through intermittent data assimilation cycles, and on the follow-on forecasts, has not been investigated in a full NWP model.

In this paper, we will discuss the development of the DPEC within the ARPS 3DVAR framework and its tests with supercell thunderstorms. The rest of the paper is organized as follows. Section 2 will discuss the schemes adopted by the 3DVAR system and focus on the development and implementation of the DPEC. Section 3 examines the impact of the DPEC on storm analysis and prediction in an OSSE framework, while section 4 applies the system to the 5 May 2007 Greensburg, Kansas, tornadic supercell thunderstorm case and examines the impact of the equation constraints on the storm forecast. The summary and future plan will be presented in section 5 .

\section{The equation constraints in the ARPS 3DVAR system}

A 3DVAR system within the ARPS model framework (Xue et al. 2000, 2001, 2003) has been developed and applied to the assimilation of weather radar and other data (Gao et al. 1999, 2004; Hu et al. 2006a,b; Hu and Xue 2007b; Xue et al. 2008; Stensrud and Gao 2010). In the system, the cost function $J$ is written as the sum of the background and observational terms plus a penalty or equation constraint term $\left(J_{c}\right)$,

$$
\begin{aligned}
J(x)= & J_{b}+J_{o}+J_{c} \\
= & \frac{1}{2}\left(\mathbf{x}-\mathbf{x}^{b}\right)^{\mathrm{T}} \mathbf{B}^{-1}\left(\mathbf{x}-\mathbf{x}^{b}\right) \\
& +\frac{1}{2}\left[H(\mathbf{x})-\mathbf{y}^{o}\right]^{\mathrm{T}} \mathbf{R}^{-1}\left[H(\mathbf{x})-\mathbf{y}^{o}\right]+J_{c} .
\end{aligned}
$$

Following the standard notion of Ide et al. (1997), $\mathbf{x}$ and $\mathbf{x}^{b}$ are the analysis and background state vectors, and $\mathbf{y}^{o}$ is the observation vector. Respectively, $\mathbf{B}$ and $\mathbf{R}$ are the background and observation error covariance matrices, and $H(\mathbf{x})$ is the nonlinear observation operator. To improve the conditioning of the $J$ minimization problem and avoid the need for the inverse of $\mathbf{B}$, a new control variable $\mathbf{v}$ is introduced, which is related to the analysis increment $\delta \mathbf{x}=\mathbf{x}-\mathbf{x}^{b}$ according to

$$
\delta \mathbf{x}=\mathbf{B}^{1 / 2} \mathbf{v} .
$$

In terms of $\mathbf{v}$, the background term becomes,

$$
J_{b}=(1 / 2) \mathbf{v}^{\mathrm{T}} \mathbf{v} .
$$

Consequently, the minimization is performed in the space of $\mathbf{v}$. The recursive filter proposed by Purser et al. (2003a,b) is used to model the effect of the background error covariance, or more precisely the square root of $\mathbf{B}$. Currently in our 3DVAR system, the background term $\mathbf{x}^{b}$ can be provided by a sounding profile, the previous ARPS model forecast, or the forecast from another model. The analysis vector $\mathbf{x}$ contains the three wind components $(u, v$, and $w)$, potential temperature $(\theta)$, pressure $(p)$, and water vapor mixing ratio $\left(q_{v}\right)$. The observations include Doppler radar radial velocity, and single- (such as surface observations) and multiple-level conventional observations (such as those of rawinsondes and wind profilers).

Term $J_{c}$ in Eq. (1) includes any penalty or equation constraint terms. Currently, it includes two terms as defined in the following:

$$
J_{c}=Q(\mathbf{x})^{\mathrm{T}} \mathbf{A}_{Q}^{-1} Q(\mathbf{x})+P(\mathbf{x})^{\mathrm{T}} \mathbf{A}_{P}^{-1} P(\mathbf{x}) .
$$

The first term on right-hand side (rhs) of Eq. (4) is intended to minimize the $3 \mathrm{D}$ anelastic mass divergence so as to provide the key coupling among the three wind components. The definition and impact of this constraint have been investigated by Gao et al. $(1999,2004)$ and $\mathrm{Hu}$ et al. (2006b). In this study, we will reinvestigate its relative impact and sensitivity to weights as compared to that of DPEC using OSSE experiments.

The second term on the rhs of Eq. (4) is the DPEC term in which

$$
\begin{aligned}
P \equiv & \nabla \cdot \mathbf{E} \\
\equiv & -\nabla^{2} p^{\prime}-\nabla \cdot(\bar{\rho} \mathbf{V} \cdot \nabla \mathbf{V}) \\
& +g \frac{\partial}{\partial z}\left[\bar{\rho}\left(\frac{\theta^{\prime}}{\bar{\theta}}-\frac{p^{\prime}}{\bar{\rho} c_{s}^{2}}+\frac{q_{v}^{\prime}}{\varepsilon+\bar{q}_{v}}-\frac{q_{v}^{\prime}+q_{\text {liquid }+ \text { ice }}}{1+\bar{q}_{v}}\right)\right] \\
& +\nabla \cdot \mathbf{C}+\nabla \cdot \mathbf{D}
\end{aligned}
$$

where

$$
\begin{aligned}
& \mathbf{E}=\frac{\partial(\bar{\rho} \mathbf{V})}{\partial t}=\mathbf{i} \frac{\partial(\bar{\rho} u)}{\partial t}+\mathbf{j} \frac{\partial(\bar{\rho} v)}{\partial t}+\mathbf{k} \frac{\partial(\bar{\rho} w)}{\partial t}, \\
& \mathbf{V}=\mathbf{i} u+\mathbf{j} v+\mathbf{k} w \\
& \mathbf{C}=\mathbf{i}(\bar{\rho} f v-\bar{\rho} \tilde{f} w)+\mathbf{j}(\bar{\rho} f u)+\mathbf{k}(\bar{\rho} \tilde{f} u) \\
& \mathbf{D}=\mathbf{i} D_{u}+\mathbf{j} D_{v}+\mathbf{k} D_{w} .
\end{aligned}
$$

The vector $\mathbf{E}$ is the forcing term of the vector Euclidian momentum equations. The primed variables are perturbations from a base state, $c_{s}$ is the acoustic wave speed, and $\varepsilon$ is the ratio of the gas constants for dry air and water vapor. The Coriolis coefficients $f=2 \Omega \sin (\phi)$ and $\tilde{f}=2 \Omega \cos (\phi)$, where $\Omega$ is the angular velocity of the earth and $\phi$ is latitude. The terms $D_{u}, D_{v}$, and $D_{w}$ contain the 

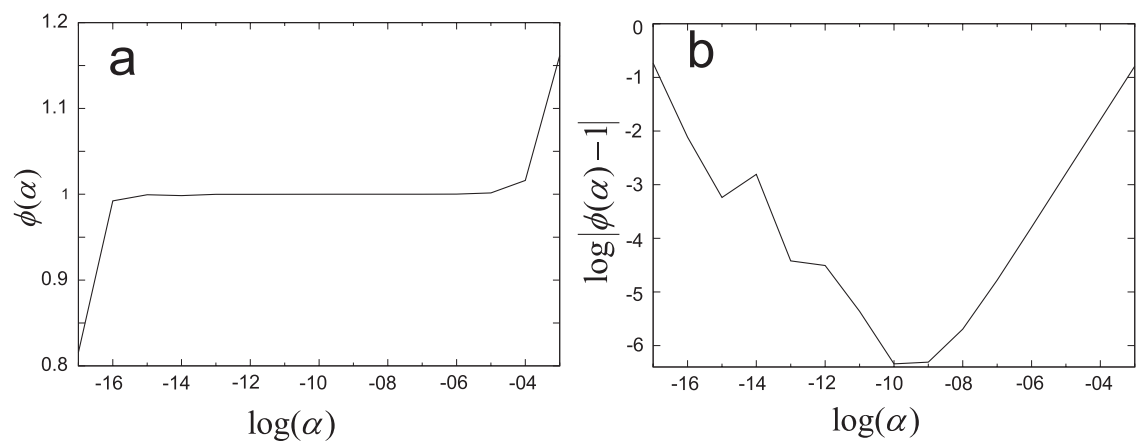

FIG. 1. The verification of the gradient calculation variation of (a) $\phi(\alpha)$ with $\log (\alpha)$ and (b) $\log |\phi(\alpha)-1|$ with $\log (\alpha)$.

subgrid-scale turbulence and computational mixing terms. Other symbols follow conventions and are detailed in the appendix. When the mass continuity equation is applied, Eq. (5) becomes $P=0$, where $P$ represents the rhs of (5).

Equation (5) is derived by applying the divergence operator to the three momentum equations of the ARPS model (Xue et al. 2000):

$$
\begin{aligned}
\bar{\rho} \frac{\partial u}{\partial t}= & -\bar{\rho} \mathbf{V} \cdot \nabla u-\frac{\partial p^{\prime}}{\partial x}+(\bar{\rho} f v-\bar{\rho} f w)+D_{u} \\
\bar{\rho} \frac{\partial v}{\partial t}= & -\bar{\rho} \mathbf{V} \cdot \nabla v-\frac{\partial p^{\prime}}{\partial y}-\bar{\rho} f u+D_{v} \\
\bar{\rho} \frac{\partial w}{\partial t}= & -\bar{\rho} \mathbf{V} \cdot \nabla w-\frac{\partial p^{\prime}}{\partial z} \\
& +\bar{\rho} g\left[\frac{\theta^{\prime}}{\bar{\theta}}-\frac{p^{\prime}}{\bar{\rho} c_{s}^{2}}+\frac{q_{v}^{\prime}}{\varepsilon+\bar{q}_{v}}-\frac{q_{v}^{\prime}+q_{\text {liquid }+ \text { ice }}}{1+\bar{q}_{v}}\right] \\
& +\bar{\rho} \tilde{f} u+D_{w^{\prime}} .
\end{aligned}
$$

Equations (10)-(12) are the basis of the thermodynamic retrieval technique mentioned in the previous section. To obtain a good storm-scale thermodynamic retrieval, it is required to have a very good estimation of the three velocity tendency terms on the left-hand side of Eqs. (10)-(12), that is, $\bar{\rho}(\partial u / \partial t), \bar{\rho}(\partial v / \partial t), \bar{\rho}(\partial w / \partial t)$. However, this task is often very difficult because the storm-scale features change very rapidly in time while radar observations are usually taken every $5-6 \mathrm{~min}$, as is the case with the U.S. operational WSR-88D radars. The inaccuracy and incompleteness of the wind observations from radars worsens the scenario. To overcome this problem and help establish better balance among model variables, we incorporate the diagnostic divergence Eq. (5) into the 3DVAR system in the form of a weak constraint [named $P$ in Eq. (4)]. In this way, the calculation of wind tendency terms is avoided.

The two As in Eq. (4) are the error covariance matrices associated with the corresponding constraints, which are assumed to be diagonal with empirically defined constant diagonal elements as the variances. The inverse diagonal matrices are called weighting coefficients and determine the relative importance of each constraint, and their optimal values can be determined through numerical experiments, similar to the way to determine certain weights in cloud-scale variation data assimilation systems (e.g., Sun and Crook 2001). Usually, the constraint terms with their weights should be similar orders of magnitude as other terms in $J$ for them to be effective.

It is very important to make sure the gradient of the cost function is computed correctly; otherwise, the minimization is erroneous. Similar to Wang (1993) and Sun et al. (1991), let $\mathbf{x}$ be the analysis vector and $J(\mathbf{x})$ be the cost function. To expand $J\left(\mathbf{x}+\alpha \nabla_{\mathbf{x}} J\right)$ at the direction $\nabla_{\mathbf{x}} J$ using the Taylor series, it can be derived that

$\Phi(\alpha)=\frac{J\left(\mathbf{x}+\alpha \boldsymbol{\nabla}_{\mathbf{x}} J\right)-J(\mathbf{x})}{\alpha \boldsymbol{\nabla}_{\mathbf{x}} J \cdot \nabla_{\mathbf{x}} J}=1.0+\mathrm{O}\left(\alpha\left\|\nabla_{\mathbf{x}} J\right\|\right)$.

For a very small $\alpha$, if the gradient is computed correctly, then the $\Phi(\alpha)$ should take the value of one. The

TABLE 1. List of OSSE data assimilation experiments (see text for details).

\begin{tabular}{lcc}
\hline \hline & $\begin{array}{c}\text { DP weighting } \\
\text { coefficient if } \\
\text { used }\end{array}$ & $\begin{array}{c}\text { MC weighting } \\
\text { coefficient if } \\
\text { used }\end{array}$ \\
\hline CNTL & $4.0 \times 10^{6}$ & $4.0 \times 10^{6}$ \\
DP & $4.0 \times 10^{6}$ & $4.0 \times 10^{6}$ \\
MC & & \\
NoEC & $8.0 \times 10^{5}$ & $4.0 \times 10^{6}$ \\
CNTLDd5 & $2.0 \times 10^{7}$ & $4.0 \times 10^{6}$ \\
CNTLDm5 & $1.6 \times 10^{5}$ & $4.0 \times 10^{6}$ \\
CNTLDd25 & $1.0 \times 10^{8}$ & $4.0 \times 10^{6}$ \\
CNTLDm25 & $4.0 \times 10^{6}$ & $8.0 \times 10^{5}$ \\
CNTLMd5 & $4.0 \times 10^{6}$ & $2.0 \times 10^{7}$ \\
CNTLMm5 & $4.0 \times 10^{6}$ & $1.6 \times 10^{5}$ \\
CNTLMd25 & $4.0 \times 10^{6}$ & $1.0 \times 10^{8}$ \\
CNTLMm25 & &
\end{tabular}



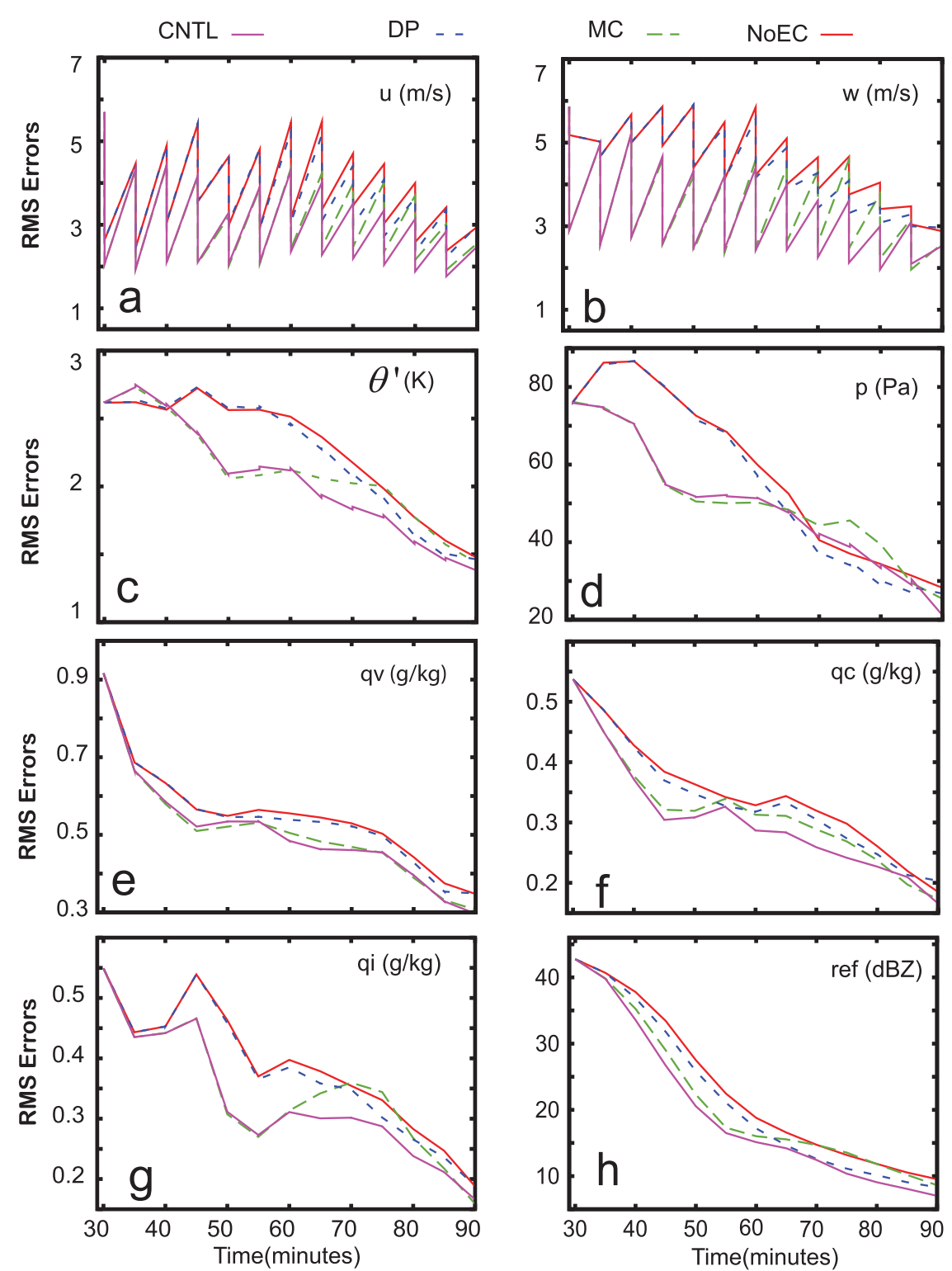

FIG. 2. The RMS errors of model fields during the 1-h assimilation period for the (a) $u$ component of wind, (b) vertical velocity $w$, (c) perturbation potential temperature, (d) pressure, (e) water vapor mixing ratio, (f) cloud water mixing ratio, (g) cloud ice mixing ratio, and (h) simulated reflectivity from model rain/snow/hail mixing ratios. CNTL (purple solid line), DP (blue dot-dashed line), MC (green dotted line), and NoEC (red solid line) are shown.

updated ARPS 3DVAR with DPEC has been verified using the above method. Figure 1 shows that when $\alpha$ takes a small value from $10^{-5}$ to $10^{-15}, \Phi(\alpha)$ takes the value of one up to the computer precision. This justifies that the gradient calculation is correct.

To check whether the minimization process goes well after including additional DPEC in the 3DVAR system, the behavior of the cost function and its individual terms are examined by plotting the evolution of the cost function with the number of iterations as similar to Gao et al. (2001). It was verified (not shown here) that the total cost function and the three individual terms (radar observation, DPEC, and MCEC, respectively) all decrease well in the minimization process for different cases and different weighting coefficients. The above tests confirm that the updated ARPS 3DVAR system with one more weak constraint (DPEC) works correctly and is ready for the following idealized testing and real case studies. 

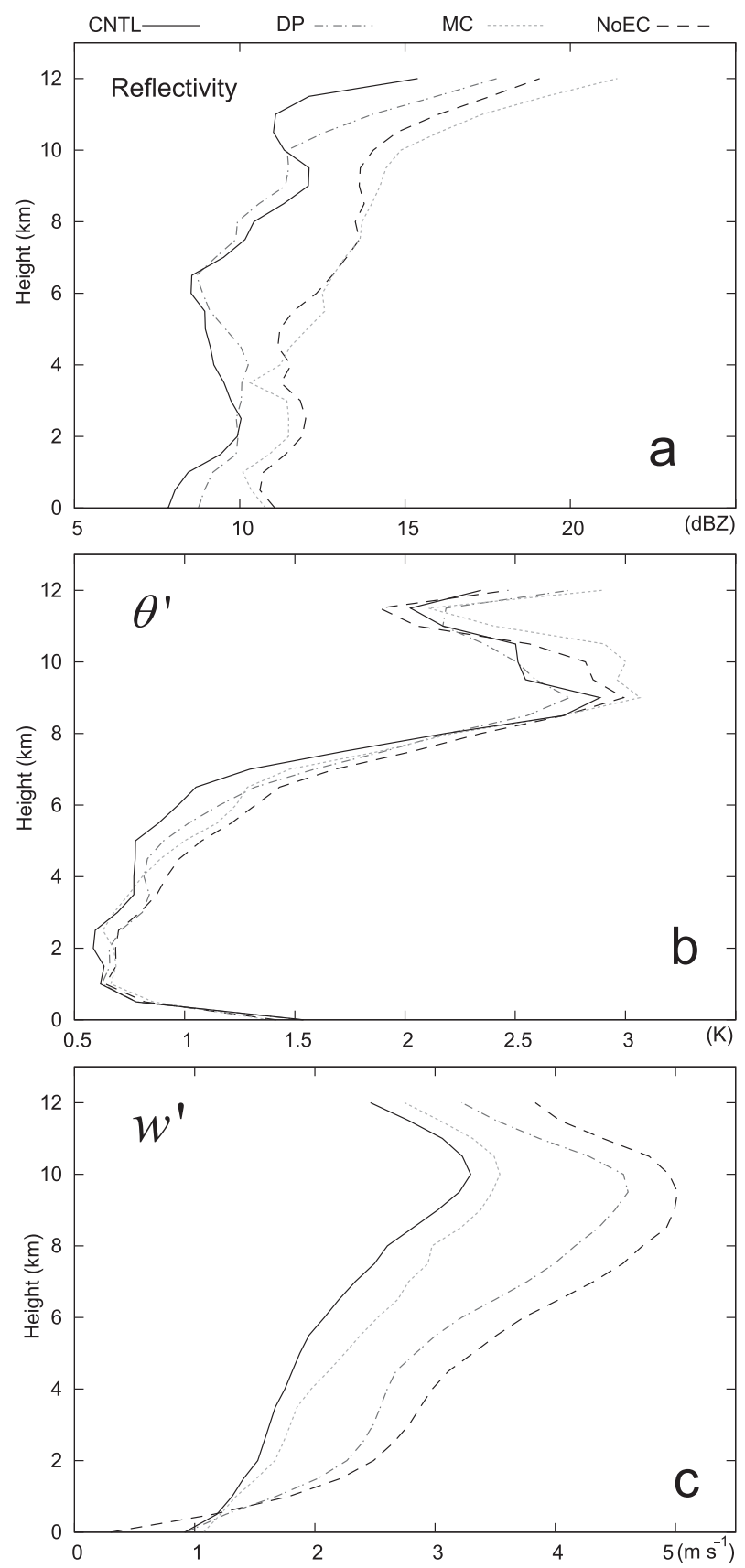

FIG. 3. The RMS error profiles after 45 min of data assimilation (nine DA cycles) for (a) reflectivity, (b) perturbation potential temperature, and (c) vertical velocity. CNTL (black solid line), DP (gray dot-dashed line), MC (gray dotted line), and NoEC (black dashed line) are shown.

\section{The impact of the diagnostic pressure equation constraint in OSSES}

\section{a. The prediction model and OSSE design}

To examine the impact of DPEC on radar data assimilation in the storm-scale 3DVAR, a series of OSSEs are conducted using simulated radar data for the 20 May 1977 Del City, Oklahoma, supercell storm (Ray et al. 1981). The ARPS model is used to create a truth simulation of the Del City supercell using a $54 \mathrm{~km} \times 54 \mathrm{~km} \times$ $16 \mathrm{~km}$ physical domain with $57 \times 57 \times 35$ grid points and $1-\mathrm{km}$ horizontal and $0.5-\mathrm{km}$ vertical resolutions, respectively. The truth simulation is initialized from a modified real sounding, as documented in Xue et al. (2001), in addition to a $+4-\mathrm{K}$ ellipsoidal thermal bubble centered at $x=48, y=16$, and $z=1.5 \mathrm{~km}$, with radii of $10 \mathrm{~km}$ in the $x$ and $y$ directions and $1.5 \mathrm{~km}$ in the $z$ direction. The Lin et al. (1983) three-ice microphysical scheme is used together with a 1.5-order turbulent kinetic energy subgrid parameterization. Open conditions are used at the lateral boundaries. A wave radiation condition is also applied at the top boundary. Free-slip conditions are applied to the bottom boundary. The length of simulation is up to $2 \mathrm{~h}$. A constant wind of $u=3 \mathrm{~m} \mathrm{~s}^{-1}$ and $v=$ $14 \mathrm{~m} \mathrm{~s}^{-1}$ is subtracted from the observed sounding to keep the primary storm cell near the center of model grid. The evolution of the simulated storms is similar to those documented in Xue et al. (2001).

In the truth simulation, the supercell rapidly develops over the first $20 \mathrm{~min}$ from the thermal bubble. The strength of the cell decreases thereafter. At around $55 \mathrm{~min}$, the cell splits into two. The north-northeastward-moving cell (the right mover) in the model tends to dominate the system. Another cell (the left mover) moves northwestward and splits again at $95 \mathrm{~min}$.

The experiments assimilate simulated radial velocity $\left(V_{r}\right)$ observations from two radars, which are located at the southwest $(x=0 \mathrm{~km}, y=0 \mathrm{~km})$ and southeast $(x=$ $54 \mathrm{~km}, y=0 \mathrm{~km}$ ) corners of the model domain. The $V_{r}$ observations are assumed to be available at the grid points and calculated as follows:

$$
V_{r}=u \sin \psi \cos \lambda+v \cos \psi \cos \lambda+w \sin \lambda,
$$

where $\psi$ is the elevation angle; $\lambda$ is the azimuth angle; and $u, v$, and $w$ are the three wind components from the truth simulation. For simplicity, the beam broadening, earth curvature effects, and the precipitation terminal velocity are not considered in either observation simulation or assimilation. Random Gaussian noise with a zero mean and a standard deviation of $1 \mathrm{~m} \mathrm{~s}^{-1}$ is added to the simulated $V_{r}$ observations.

The first 3DVAR analysis assimilates radar data taken from truth simulation at $t=30 \mathrm{~min}$ of model time and its first guess is horizontally homogeneous, with vertical variation specified by the same sounding profile as the truth simulation, which means there is no storm in the background at this time. From the first analysis, the ARPS model is run for 5 min when another 3DVAR analysis 

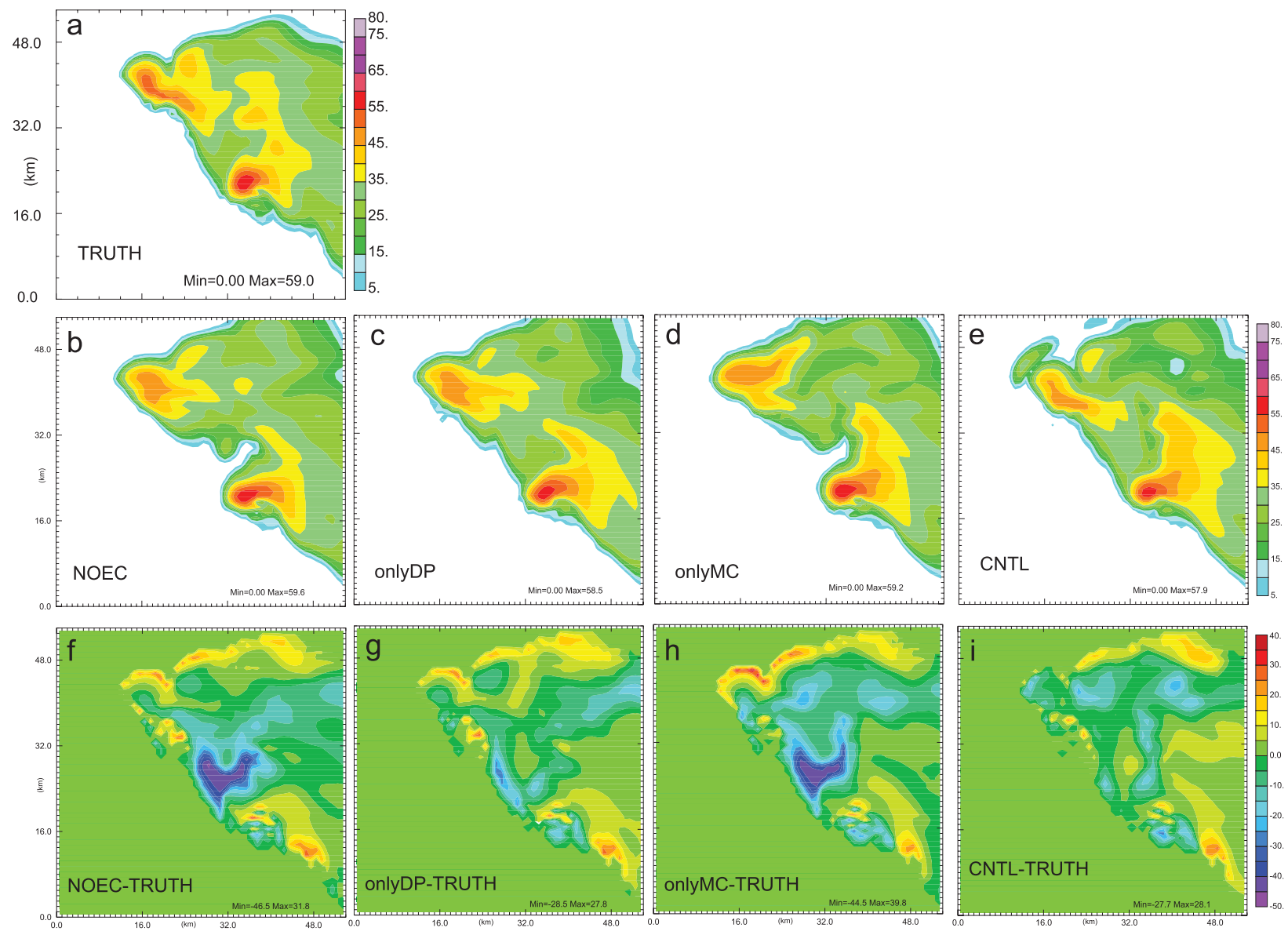

FIG. 4. The simulated reflectivity fields at $z=6.5 \mathrm{~km}$ MSL for (a) the truth simulation experiments (b) NoEC, (c) DP, (d) MC, and (e) CNTL; and the differences of the analyzed reflectivity from the truth at the same level for (f) NoEC, (g) DP, (h) MC, and (i) CNTL. All fields are at 75-min model time or after 45 min of data assimilation.

is performed using the forecast as the background. Such intermittent assimilation cycles are repeated every 5 min until $90 \mathrm{~min}$, giving a data assimilation period of $60 \mathrm{~min}$

Table 1 lists all of the data assimilation experiments. The first four experiments (CNTL, MC, DP, and NoEC) are designed to investigate the impact of DPEC. For comparison purposes, the impact of MCEC is also examined. Experiment CNTL includes both constraints DPEC and MCEC. Experiment MC includes MCEC only, while experiment DP includes DPEC only. In experiment NoEC, neither DPEC nor MCEC is included.

The next four experiments, CNTLDm5, CNTLDd5, CNTLDm25, and CNTLDd25, are designed to test the sensitivity of the data assimilation to the DPEC weighting coefficient. CNTLDm5 is the same as CNTL except that the DP weighting coefficient used in CNTL is multiplied by 5. In CNTLDd5, the DP weighting coefficient is divided by 5 . In CNTLDm 25 and CNTLDd25, the coefficients are changed by a factor of 25 .
The next four experiments, CNTLMm5, CNTLMd5, CNTLMm25, and CNTLMd25, are designed to test the sensitivity to the MCEC weighting coefficient. They are the same as CNTL except that the MCEC weight is changed by a factor of 5 or 25 , following the same naming convention as the DPEC sensitivity experiments.

To compare the accuracy of the data assimilation results from different experiments, the root-mean-squared (RMS) error statistics of model variables between the experiments and the truth simulation are computed as

$$
\text { RMS_s }=\sqrt{\frac{1}{N} \sum_{i=1}^{N}\left(s-s_{t}\right)_{i}^{2}},
$$

where $N$ is the total number of $3 \mathrm{D}$ grid points used in the calculation, and the subscript $t$ stands for the truth simulation. The computation of the RMS error statistics is only done over model grid points where the reflectivity (calculated from the local hydrometeor mixing ratios) of 

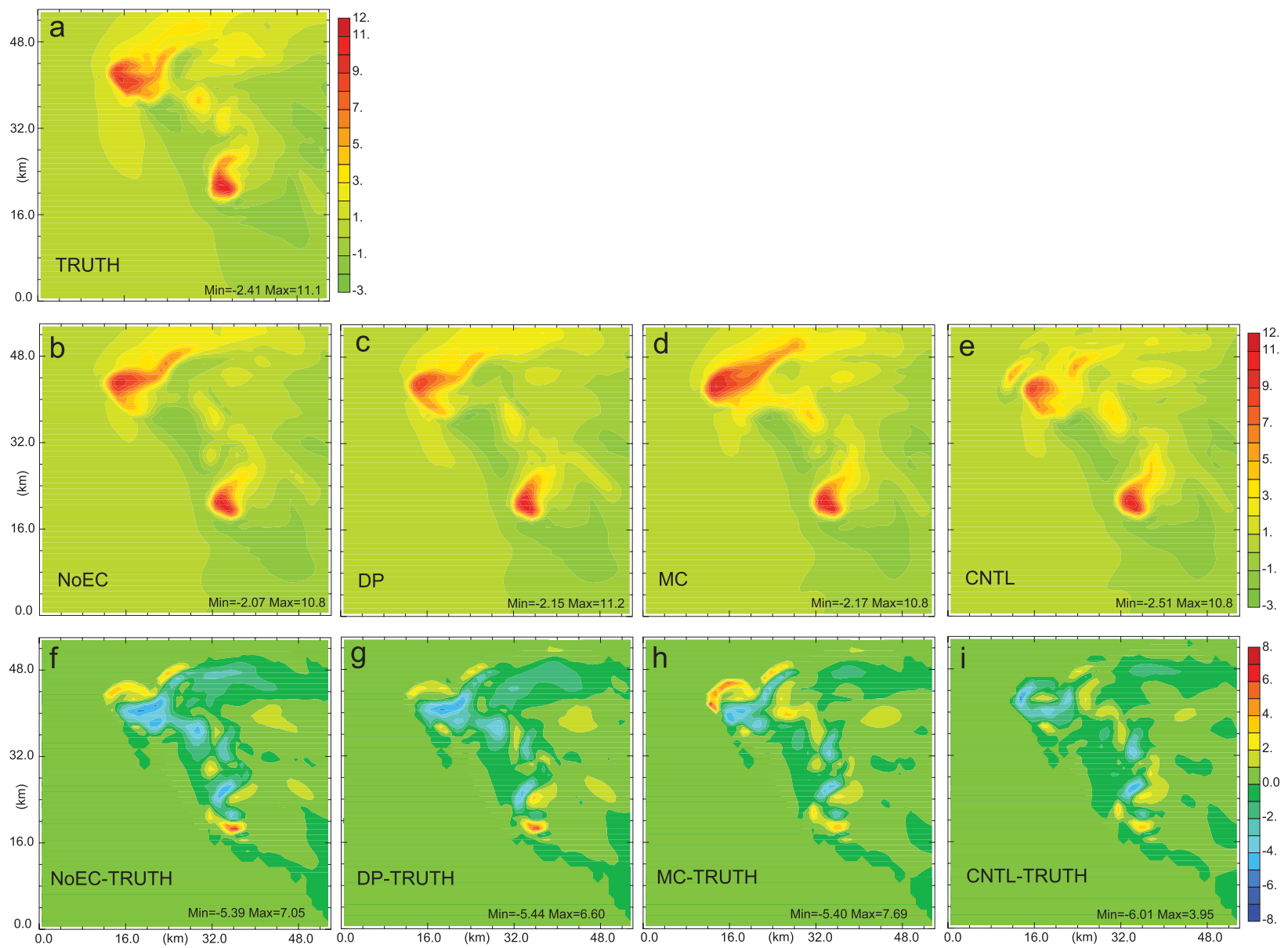

FIG. 5. The perturbation potential temperature at $z=6.5 \mathrm{~km}$ MSL for (a) the truth simulation, (b) NoEC, (c) DP, (e) MC, and (e) CNTL; and the difference of the temperature field between each data assimilation experiment and the truth simulation at $z=6.5 \mathrm{~km}$ MSL for (f) NoEC, (g) DP, (h) MC, and (i) CNTL. All the above plots are available at $t=75$ min into truth simulation (i.e., after 45-min data assimilation).

the truth simulation is greater than $10 \mathrm{~dB} Z$, which roughly corresponds to storm region.

\section{b. Results of OSSES}

To investigate the impact of DPEC, the RMS error statistics are calculated during the whole assimilation period for model variables, as shown in Fig. 2. For ease of display, the RMS errors of simulated reflectivity are shown in place of the errors for rain/snow/hail mixing ratios, and the RMS error for the $v$ wind component is not shown, because it evolves similarly to the $u$ wind component.

From Fig. 2, it is seen that the errors are the lowest in CNTL, where both DPEC and MCEC are included. When only MCEC is included in MC, the errors are also lower than those of NoEC and DP, but somewhat higher than those of CNTL, especially during the earlier cycles. Including DPEC only is less effective than including
MCEC only during the earlier cycles, but its accumulated effects seem to exceed those of MCEC during the later cycles in MC, especially for temperature and pressure, which are most directly involved in the diagnostic pressure equation (Figs. 1c,d). These results indicate that including both DPEC and MCEC yields the best results and the addition of DPEC has positive impacts.

To more clearly illustrate the impact of the two constraints, Fig. 3 shows the RMS error profiles after $45 \mathrm{~min}$ of data assimilation (nine DA cycles). The RMS error of reflectivity is evidently reduced in DP compared to NoEC and in CNTL compared to MC, both of which are due to the addition of DPEC. The largest difference in reflectivity is over $4 \mathrm{dBZ}$ between 6- and 8-km levels. At most levels, the RMS errors of CNTL that includes both constraints are the smallest among all four experiments. For potential temperature, DP and CNTL generally produce smaller RMS error than NoEC and MC, respectively, 

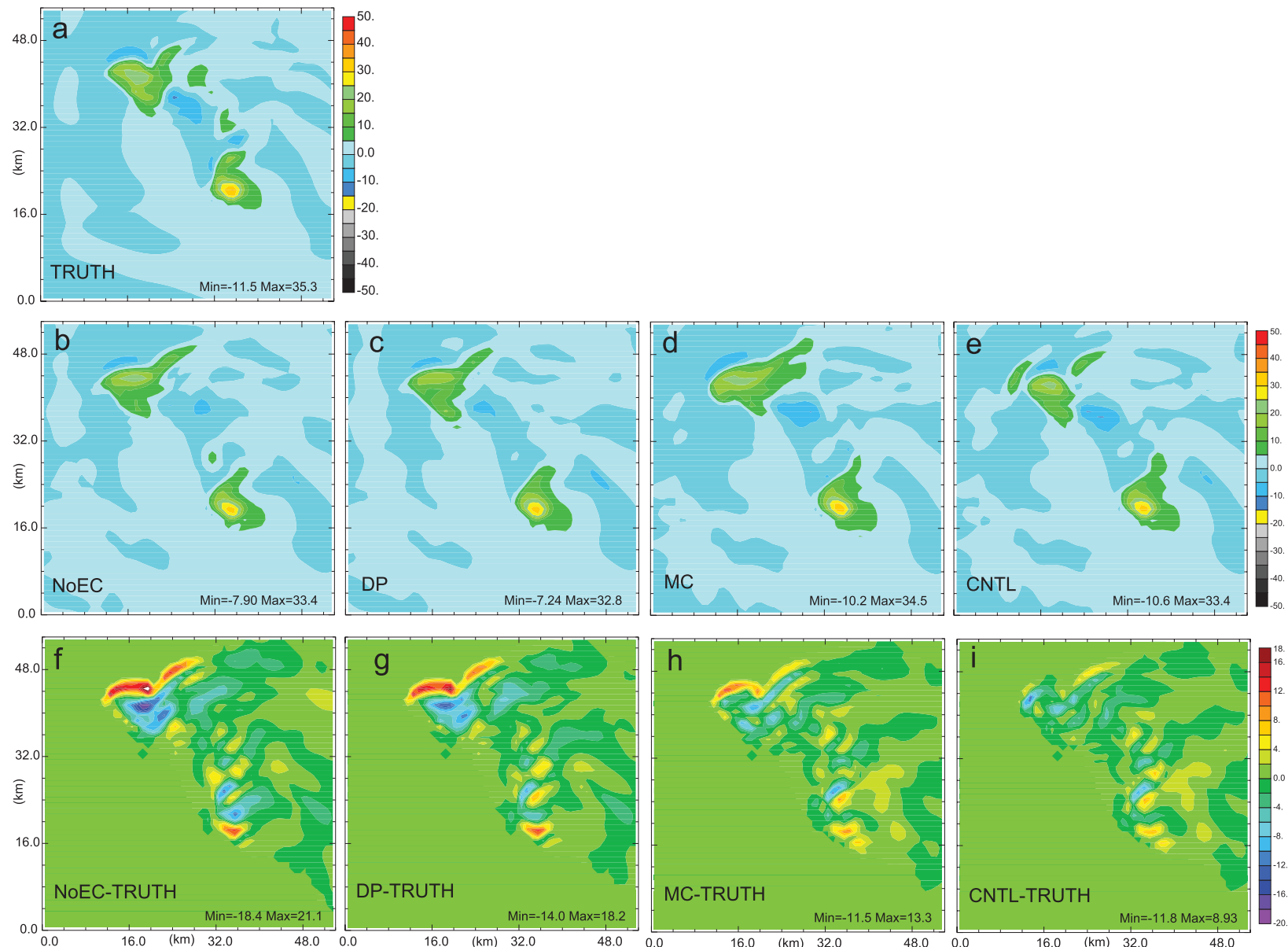

FIG. 6. The vertical velocity at $z=6.0 \mathrm{~km}$ MSL for (a) the truth simulation, (b) CNTL, (d) NoEC, (e) DP, (f) MC; and the difference of the vertical velocity between each data assimilation experiment and the truth simulation at $z=6.0 \mathrm{~km} \mathrm{MSL} \mathrm{for} \mathrm{(c)} \mathrm{CNTL,} \mathrm{(g)} \mathrm{NoEC,}$ (h) DP, and (i) MC. All the above plots are available at $t=75$ min into truth simulation (i.e., after 45-min data assimilation).

with noticeable differences between 4 and $6.5 \mathrm{~km}$, and between 8.5 and $12 \mathrm{~km}$ (Fig. 3b). Figure 3c shows that the additional use of DPEC in DP reduces the RMS error of vertical velocity by about $0.4 \mathrm{~m} \mathrm{~s}^{-1}$ at $10 \mathrm{~km}$ MSL compared to NoEC, and similarly between CNTL and MC (Fig. 3c). The use of MCEC reduces the $w$ error by over $1 \mathrm{~m} \mathrm{~s}^{-1}$ near the $10-\mathrm{km}$ level as shown by comparing $\mathrm{MC}$ and NoEC. The use of both equation constraints (CNTL) produces an error reduction of about $1.6 \mathrm{~m} \mathrm{~s}^{-1}$ compared to the NoEC case at the same level. The decrease in the vertical velocity RMS error by the inclusion of equation constraints is very obvious above the $1.5-\mathrm{km}$ level.

To see the differences in the analyzed model fields, we show in Figs. 3-5 the analyzed reflectivity, $\theta^{\prime}$ and $w$ fields, and their differences from the truth, together with the truth fields themselves, from the four experiments at 75 min of truth simulation time or 45 min into the data assimilation. Figure 4 shows the reflectivity fields at $6.5 \mathrm{~km}$
MSL. At this time, the reflectivity pattern for the rightmoving cell is recovered well in all four experiments. Larger errors lie with the left-moving cell in the northwestern part of the domain and in the region between the two cells, especially in NoEC and MC (Figs. 4f,h). Experiments DP and CNTL, with the inclusion of DPEC, are seen to produce better analyses of reflectivity than NoEC and MC (cf. Figs. 4g, i versus Figs. 4f,h). Experiment CNTL yields the best results in terms of the reflectivity error in the whole domain (Fig. 4i).

Figure 5 shows the corresponding analyzed $\theta^{\prime}$ fields and their differences from the truth. In general, the $\theta^{\prime}$ field is recovered very well for both left- and right-moving cells in all four experiments, and the pattern differences among the experiments are relatively small at first glance. However, from the difference fields, it can be clearly seen that the use of DPEC only in DP mainly helps improve the recovery of the temperature structure with the left-moving cell (cf. Figs. 5g,f), and MCEC only in MC mainly helps 

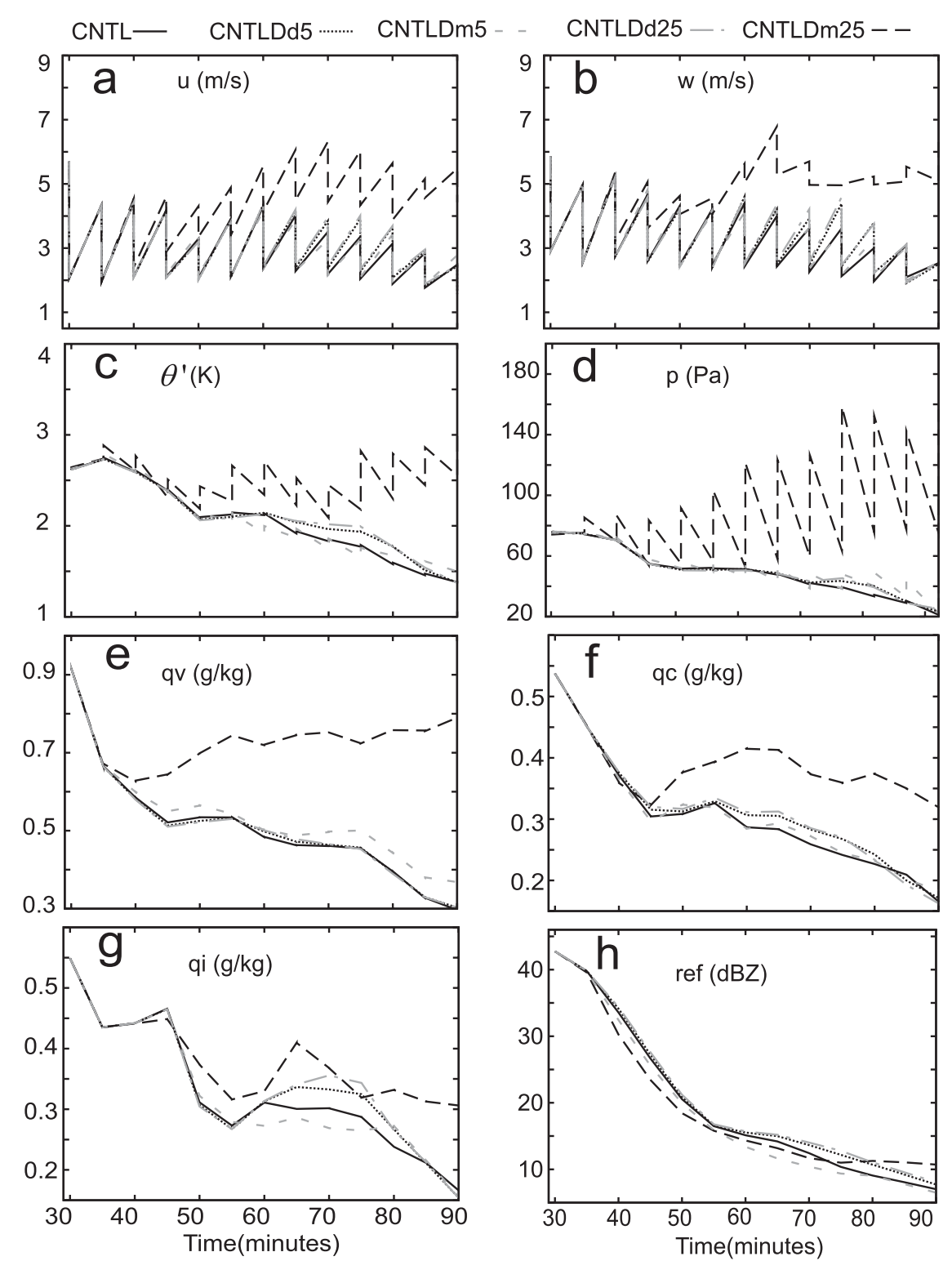

FIG. 7. The RMS error of model fields during the 1-h assimilation period for the (a) $u$ component of wind, (b) vertical velocity $w$, (c) perturbation potential temperature, (d) pressure, (e) water vapor mixing ratio, (f) cloud water mixing ratio, (g) cloud ice mixing ratio, and (h) simulated reflectivity from model rain/snow/hail mixing ratios. CNTL (black solid line), CNTLDd5 (black dotted line), CNTLDm5 (gray short-dashed line), CNTLDd25 (gray dot-dashed line), and CNTLDm25 (black long-dashed line) are shown.

improve the temperature structure with the right-moving cell (cf. Figs. 5h,f). As a whole, experiment CNTL with both constraints again performs the best in terms of the analyzed temperature structures for the whole storm system, given the generally smaller temperature errors (Fig. 5i).

In Fig. 6, the corresponding $w$ fields at $z=6 \mathrm{~km} \mathrm{MSL}$ are shown. It is easily seen that the $w$ structure associated with the right-moving storm cell is recovered well at this time in all four experiments (Figs. 6b-e). For the left-moving cell there are larger differences. Overall, CNTL produces the best analysis of $w$ field, and MC the second best. Comparing the error fields, it is clear that MCEC has a larger impact on the $w$ analysis than DPEC, which is not surprising because the mass continuity equation is mostly responsible for linking $w$ with the horizontal velocity components, which are much better observed by the radars.

To summarize, both DPEC and MCEC built into the 3DVAR provide a positive impact on the analysis of 

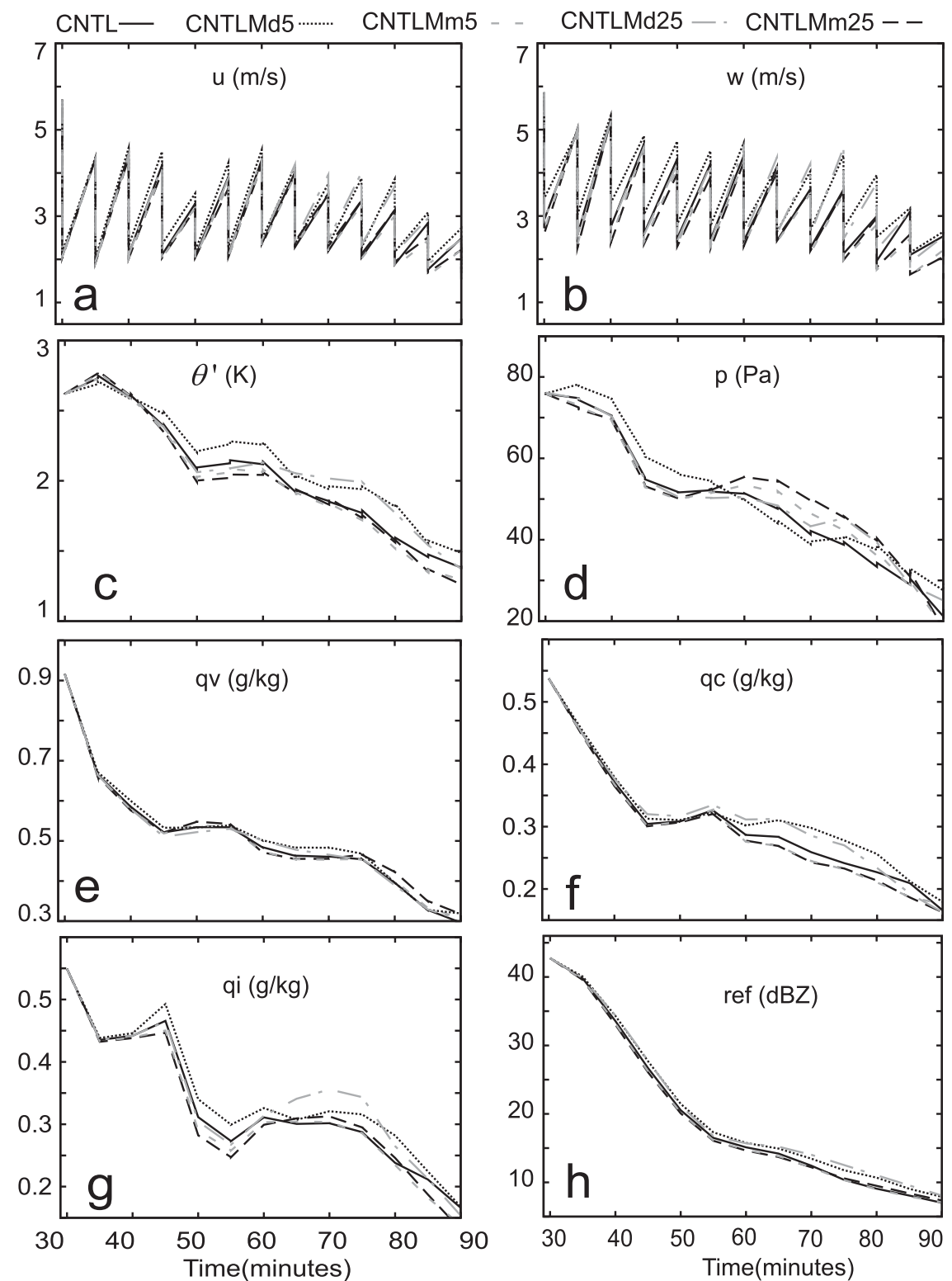

FIG. 8. The evolution of RMS error of model fields during the 1-h assimilation period for (a) $u$ component of wind fields, (b) vertical velocity, (c) potential temperature, (d) pressure, (e) water vapor mixing ratio, (f) cloud water mixing ratio, (g) cloud ice mixing ratio, and (h) simulated reflectivity from model rain/snow/hail mixing ratio. CNTL (black solid line), CNTLMd5 (black dotted line), CNTLMm5 experiment (gray short-dashed line), CNTLMd25 experiment (gray dot-dashed line), and CNTLMm25 experiment (black long-dashed line) are shown.

a supercell storm system when assimilating radar radial velocity data through intermittent assimilation cycles. The impact of MCEC is clearer at the first several data assimilation cycles, while the impact of DPEC is more obvious in later cycles. Including both constraints at the same time yields the best data assimilation results in most model variables, especially for $w$ and $\theta$, the hydrometeor fields as represented by simulated reflectivity.
To test the sensitivity of the results to the weighting coefficients of DPEC and MCEC, eight more sensitivity experiments are conducted (Table 1). These experiments are similar to CNTL, which uses both constraints, except for the different values of their weight coefficients. Figures 7 and 8 show the evolution of the RMS errors of these sensitivity experiments, as well as CNTL during the 1-h data assimilation period for various model fields. 


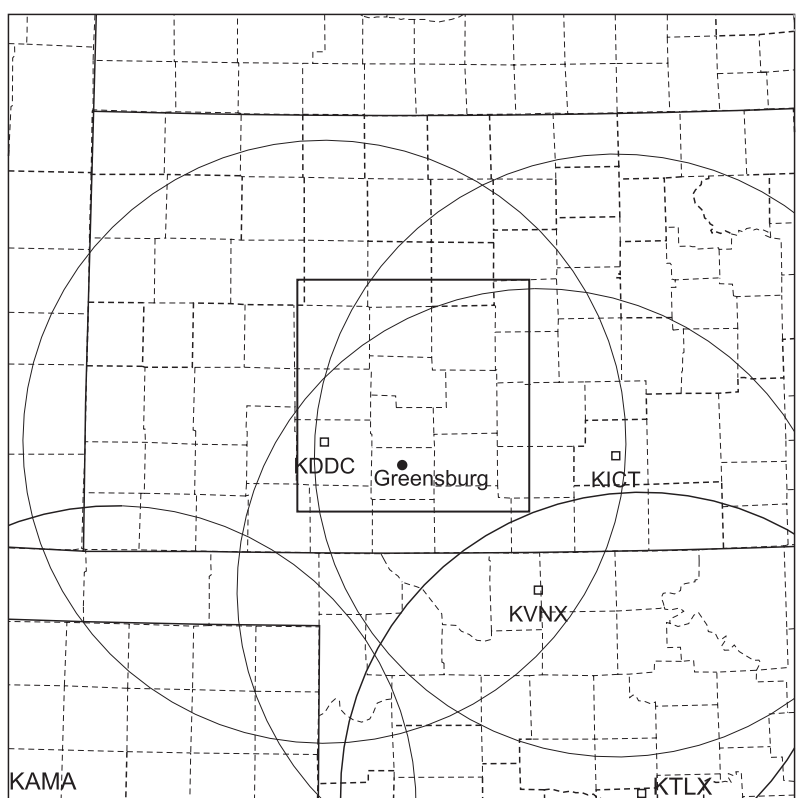

FIG. 9. The model domain with county boundaries for the 5 May 2007 Greensburg tornadic supercell thunderstorm case study. The five radars as well as their $230-\mathrm{km}$ range circles are also shown. The location of the town of Greensburg (black dot) is shown, as is the domain coverage (black bold inner box) in Fig. 10.

The sensitivity to the DPEC weighting coefficient is shown in Fig. 7. When the DPEC weighting coefficient is increased or decreased by a factor of 5 , the RMS errors are not changed much compared to those of CNTL for all model variables. When the DPEC weighting coefficient is decreased by a factor of 25 , the RMS errors are still not too different. However, when the DPEC weighting coefficient is increased by a factor of 25 , the RMS errors become significantly larger than those of CNTL, especially for $u, w$, pressure, and $\theta$; their errors even increase with the data assimilation cycles (Figs. 7a-d). This indicates that one should be cautious in choosing the DPEC weighting coefficient. A very large weighting coefficient should be avoided, because it might give DPEC much more of the total cost function and then degrade the quality of the data assimilation.

Figure 8 presents the sensitivity to the MCEC weighting coefficient. When the weighting coefficient is increased or decreased by a factor of 5, the RMS errors are not changed much. When the weighting coefficient is changed by a factor of 25 , the RMS errors are increased somewhat in later assimilation cycles for temperature, pressure, and cloud water and ice mixing ratios (Figs. 8d-g). Therefore, it can be concluded that the mass continuity equation constraint is not very sensitive to the weighting coefficient within reasonable range.

In summary, in this section, we demonstrated the positive impact of DPEC and MCEC in the 3DVAR system with simulated radar data. It was found that the data assimilation results are the best when both constraints are utilized. In next section, we examine the impact of DPEC using a real case.

\section{The 5 May 2007 Greensburg tornadic supercell storm case}

The 5 May 2007 Greensburg, Kansas, tornadic thunderstorm complex produced 18 tornadoes in the Dodge City forecast area and 47 tornado reports in Kansas, Nebraska, and Missouri. One of them is the strongest tornadoes in recent years. This tornado started moving through Greensburg at 0245 UTC 5 May 2007 [2145 central daylight time CDT) 4 May] and destroyed over $90 \%$ of the town. The tornado damage was rated at EF5-the highest rating on the enhanced Fujita scale (McCarthy et al. 2007). A detail description of the supercell that spawned this tornado and its environment setting can be found in Stensrud and Gao (2010).

For this real data case, we use 3-km grid spacing with $200 \times 200$ grid points in the horizontal. The ARPS model domain is shown in Fig. 9. The domain is selected with sufficient coverage to contain the principal features of interest while maintaining some distance between the primary storms and the lateral boundaries. The model uses 47 terrain-following vertical layers, with nonlinear vertical stretching, via a hyperbolic tangent function that yields a spacing of $100 \mathrm{~m}$ at the ground and expands to approximately $800 \mathrm{~m}$ at the top of the domain. The Lin et al. (1983) three-ice microphysical scheme is used together with a 1.5-order turbulent kinetic energy subgrid parameterization.

The impact of the DPEC will be discussed in terms of the quality of ensuing forecasts resulting from the analysis because no truth analysis is available for verification. Two experiments are conducted. The first experiment does not include DPEC in $J$ and will be referred as experiment NoDPr (" $r$ " incidates real case). The second experiment uses DPEC with the DP weighting coefficient of 1.0E8 and is referred as experiment CNTLr. In both experiments the mass continuity equation constraint is used with the $\mathrm{MC}$ weighting coefficient of $1.0 \times 10^{8}$.

For both experiments, data from five radars at Dodge City, Kansas (KDDC); Vance Air Force Base, Oklahoma (KVNX); Wichita, Kansas (KICT); Oklahoma City, Oklahoma (KTLX); Amarillo, Texas (KAMA) are used (Fig. 8). The initial analysis background and the boundary conditions come from the mean of a mesoscale ensemble assimilation system run at $30-\mathrm{km}$ grid spacing (Stensrud and Gao 2010). As for the idealized case, only radial velocity data are used. While Stensrud and Gao (2010) performed a 3DVAR analysis only at one time 

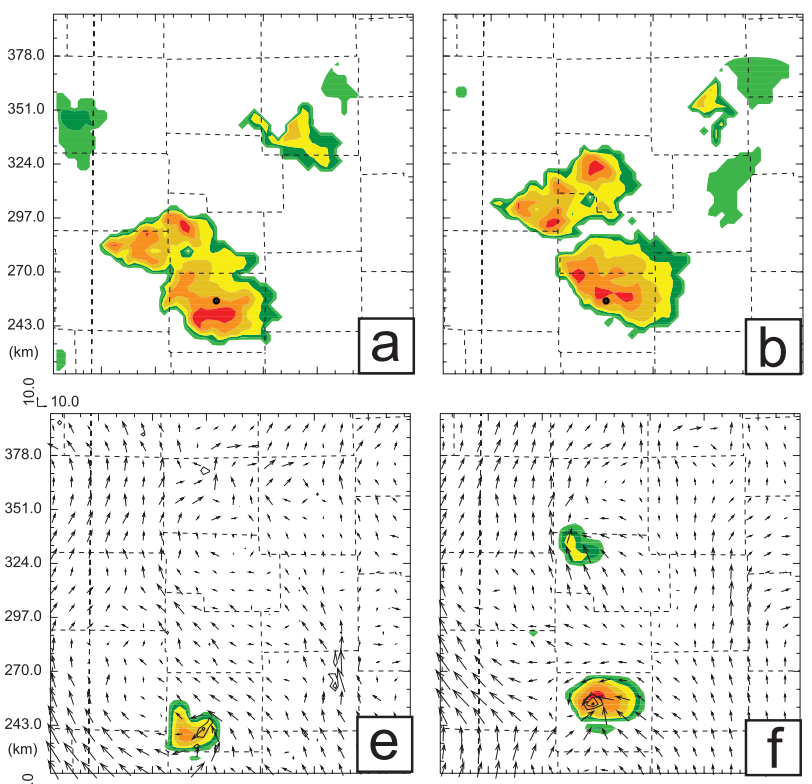

$\stackrel{\circ}{\circ} 10.0$
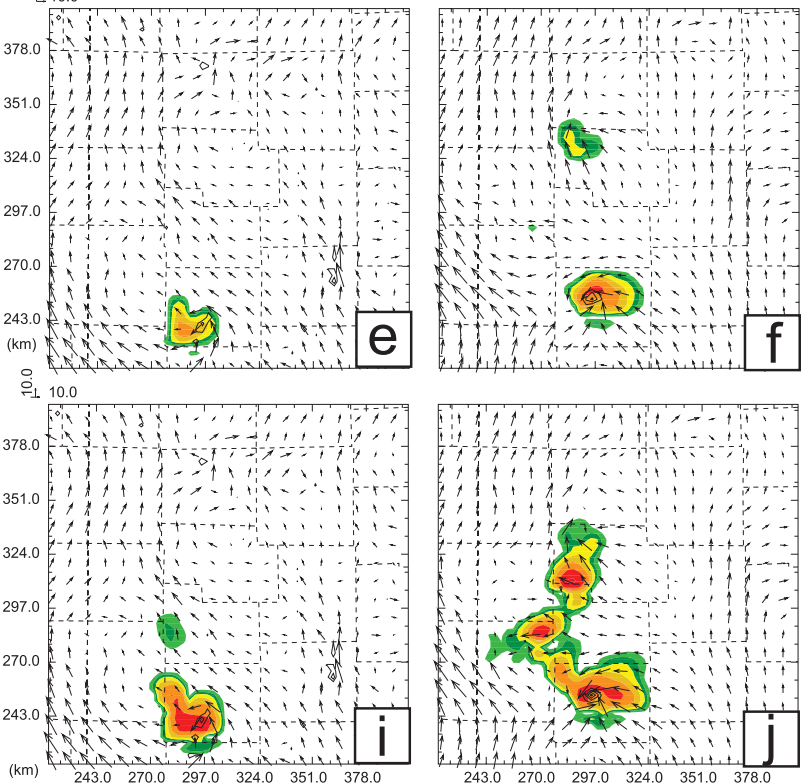
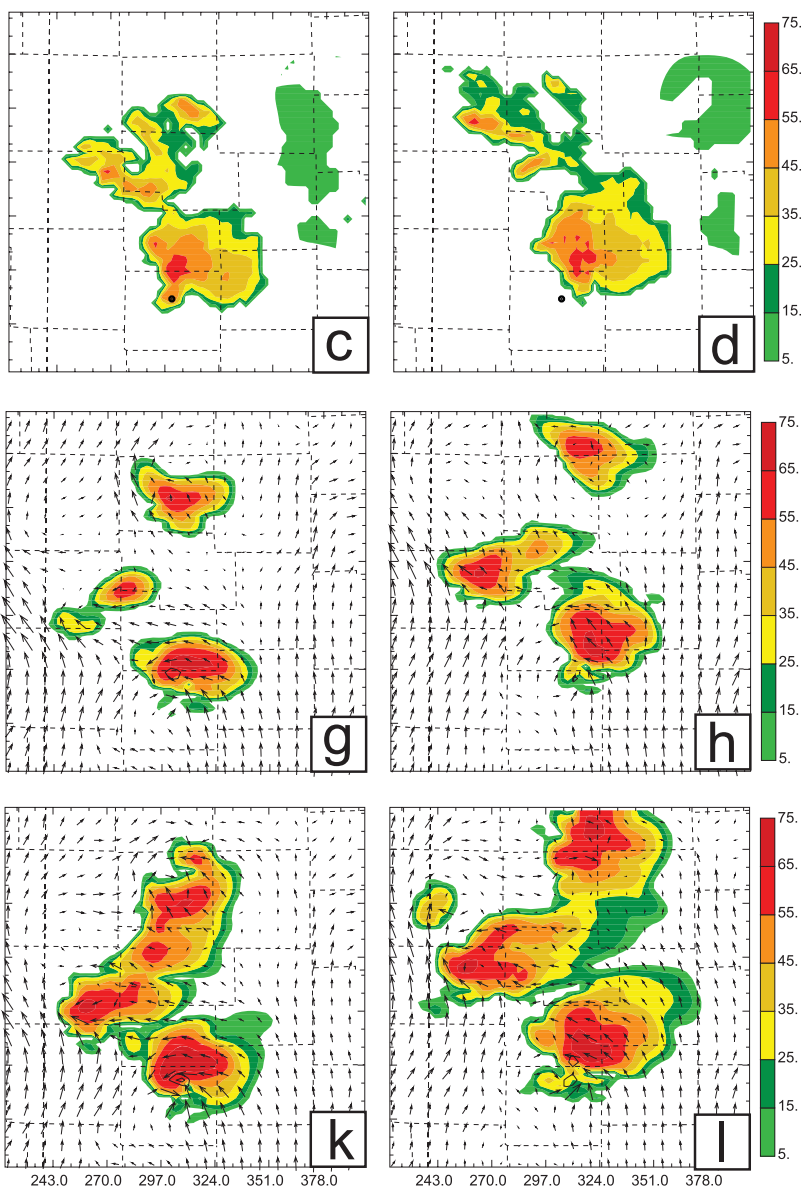

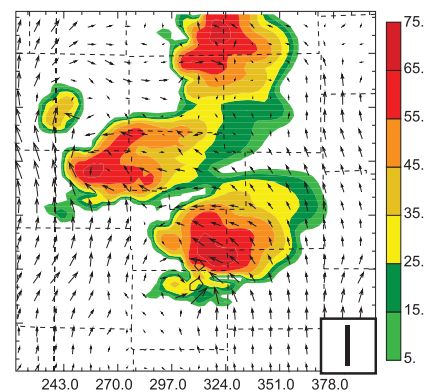

FIG. 10. Observed radar reflectivity mosaic (dBZ) at 2-km MSL from KDDC, KICT, KVNX, KAMA, KTLX Doppler radars valid at (a) 0230, (b) 0250, (c) 0310, and (d) 0330 UTC; simulated radar reflectivity (dBZ); horizontal winds; and vertical vorticity (contours staring at $0.005 \mathrm{~s}^{-1}$ with an interval of $0.005 \mathrm{~s}^{-1}$ ) at $2 \mathrm{~km} \mathrm{MSL}$ from NoDPr valid at (e) 0230, (f) 0250, (g) 0310, and (h) 0330 UTC; and from CNTLr valid at (i) 0230, (j) 0250, (k) 0310, and (l) 0330 UTC. The duration 0230-0330 UTC covers the 1-h forecast period. (a)-(d) The location of the town of Greensburg (black dots) is shown.

level before the launch of the forecast, the present study performs cycled 3DVAR analyses with a 1-h-long assimilation period before the forecast. A 5-min ARPS forecast follows each analysis, and this process is repeated until the end of the 1 -h assimilation period. From the final analysis, a 1-h forecast is launched. Thus, each experiment consisted of a 1-h assimilation period (from 0130 to 0230 UTC) and a 1-h forecast period (0230-0330 UTC).

The radar reflectivity mosaic from the aforementioned five WSR-88D radars is used for forecast verification. The evolution of the storm as indicated by the radar reflectivity mosaic at $2 \mathrm{~km}$ MSL is shown in Fig. 10 from 0230 to 0330 UTC every $20 \mathrm{~min}$. We focus on the discussion of the major supercell thunderstorm at the southernmost side that produces the EF-5 tornado hitting the Greensburg area between 0245 and $\sim 0305$ UTC. It bears a hook echo signature at 0230 UTC (Fig. 10a). As the major storm reaches Greensburg, the hook echo signature becomes less prominent (Figs. 10b,c) due to reflectivity wrap up. During this period, the radar velocity observations (not shown) indicate strong cyclonic rotation associated with the strong tornado. The major storm moves gradually toward northeast. After passing the town of Greensburg, the storm maintains a visible hook echo and continues to move to the northeast. A second EF-3 tornado develops at the end of Greensburg tornado just northeast of the town (McCarthy et al. 2007).

Similar to the idealized case in section 3, the impact of DPEC at the first several cycles is not obvious. There are minimal pattern or feature differences in the analyzed fields during the earlier cycles (not shown). However, after 1-h data assimilation, the impact of DPEC looks clearer. In CNTLr (Fig. 10i), the major storm is more prominent in NoDPr (Fig. 10e), bearing stronger reflectivity and covering a broader area at the end of the 1-h data assimilation, comparing better to the reflectivity 


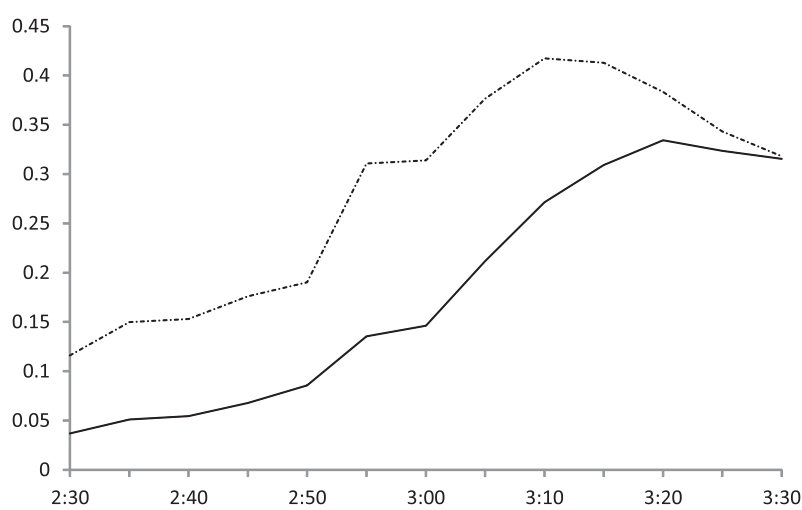

FIG. 11. Equitable threat scores of predicted reflectivity at $2.1 \mathrm{~km}$ MSL with $15-\mathrm{dBZ}$ thresholds. NoDPr (solid line) and CNTLr (dashed line) are shown.

observation (Fig. 10a). North of the major storm, a storm cell also develops in CNTLr (Fig. 10i), although it is much weaker than observed (Fig. 10a). At the upper levels, this cell is better developed (not shown). In comparison, NoDPr completely misses this cell at this time. Based on these results, we can say that the inclusion of DPEC does help improve the storm analysis, consistent with our earlier OSSE results.

From the improved analysis, a better forecast should be expected. Forecasts are launched from each of the above two analyses. After $20 \mathrm{~min}$ of forecast at 0250 UTC, the major storm cell in CNTLr strengthens and develops a hook echo signature with a clearly defined vorticity center while two more cells are fully developed to its north and northwest (Fig. 10j), more or less matching the observation (Fig. 10b). On the other hand, the primary cell in NoDPr is weaker and the matching northwest cells are completely absent (Fig. 10f). During the next $20 \mathrm{~min}$ until 0310 UTC, the primary cell in CNTLr develops further and shows a clearer hook echo signature (Fig. $10 \mathrm{k})$. Overall, the intensity of the cells is overpredicted, which may have to do with model error or error in the storm environment. Stensrud and Gao (2010) found strong sensitivity of the forecast to the storm environment, while Dawson et al. (2010) had shown the large sensitivity of predicted storm to microphysics parameterization. The cells in NoDPr are generally weaker (Fig. 10g), but do not necessarily show a better match to the observation (Fig. 10c). By the end of the 1-h forecast period, the major storm is predicted well in both CNTLr and NoDPr in terms of its moving path and general rainfall coverage. Both experiments overpredict the northern cells. To quantitatively evaluate the above two forecasts, equitable threat scores (ETSs) for reflectivity at $2.1 \mathrm{~km} \mathrm{MSL}$ (with the 15-dBZ threshold) are computed and plotted in Fig. 11. It is seen that CNTLr (dashed line) yields

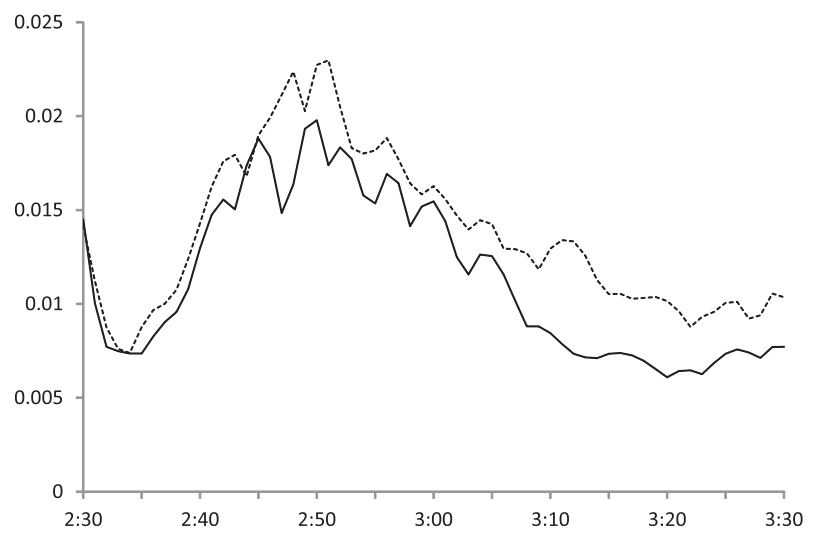

FIG. 12. The time series of maximum vertical vorticities below $2 \mathrm{~km}$ from 0230 to 0330 UTC 5 May 2007 every $1 \mathrm{~min}$. The horizontal axis shows the time (UTC), the vertical axis shows the vertical vorticity value $\left(\mathrm{s}^{-1}\right)$. Experiments NoDPr (solid line) and CNTLr (dashed line) are shown.

higher scores than NoDPr (solid line), which agrees with our subjective assessment above.

To further examine the difference in the two forecasts, the maximum vertical vorticity $(\zeta)$ below $2 \mathrm{~km}$ MSL is computed from the forecasts every minute. The time series of the $\zeta$ are plotted in Fig. 12. It is shown that CNTLr (dashed line) predicts larger low-level maximum $\zeta$ than NoDPr (solid line) during the entire forecast period, especially during the last half-hour. A larger lowlevel $\zeta$ usually indicates stronger and deeper mesocyclone rotation. As an example, Fig. 13 shows $\zeta$ in a vertical cross section through the low-level maximum $\zeta$ center of the major storm at $y=253.5 \mathrm{~km}$ at 0250 UTC for both experiments. It can be seen that CNTLr (Fig. 13b) predicts a stronger and deeper rotating column than NoDPr (Fig. 13a). Considering that this is a strong tornadic supercell, we believe the forecast of CNTLr is more accurate.

\section{Summary and conclusions}

Storm-scale 3DVAR is computationally efficient and operationally feasible for assimilating full-volume Doppler radar data for the prediction of thunderstorms. However, it is often challenged as being less optimal for the stormscale application because of its use of static background error covariance and lack of balance among analyzed model variables. To reduce this problem, we proposed to incorporate into the ARPS 3DVAR cost function a weak constraint based on the diagnostic pressure equation derived from the full ARPS momentum equations. The main goal is to help improve dynamic consistency among model variables and therefore improve the analysis of convective storms and their subsequent forecast. 

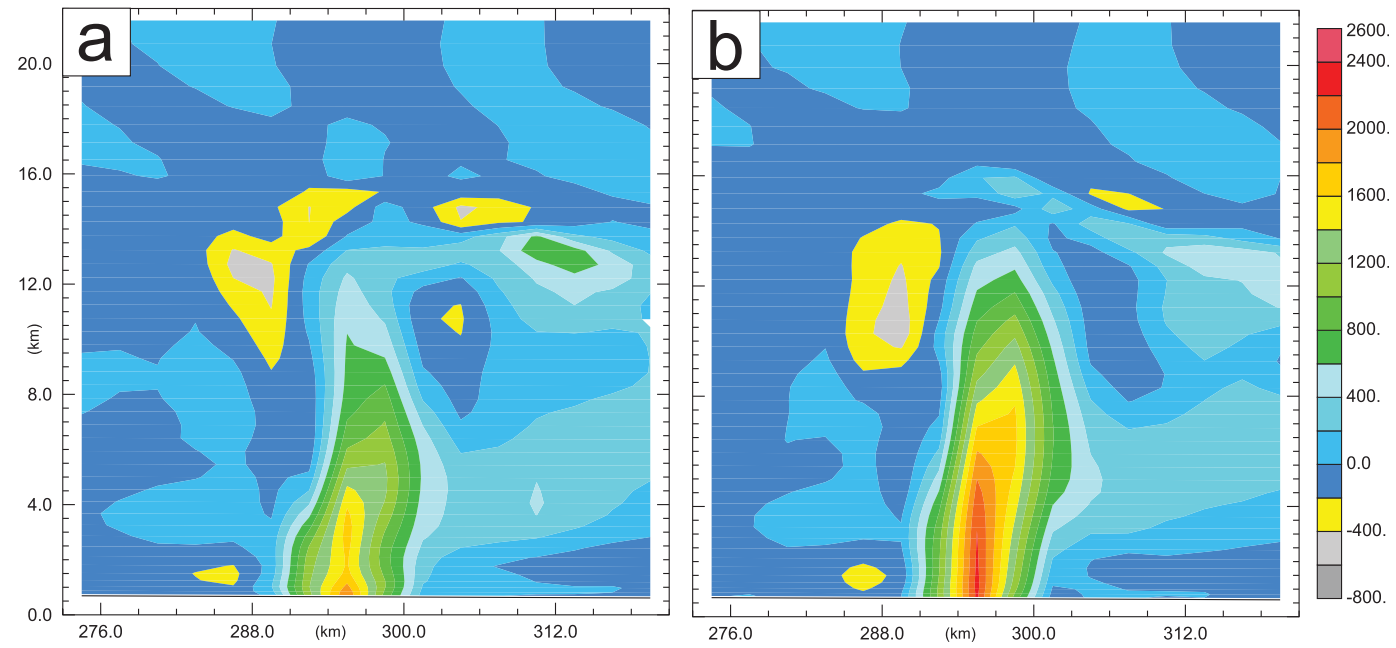

FIG. 13. The vertical vorticity $\left(10-5 \mathrm{~s}^{-1}\right)$ at the vertical cross section through the center of the major storm at $y=253.5 \mathrm{~km}$ at 0250 UTC 5 May 2007 for the (a) NoDPr and (b) CNTLr.

The impact of DPEC is first investigated using an idealized tornadic supercell thunderstorm through OSSEs. A 1-h data assimilation period is used through 5-min intermittent assimilation cycles and radar radial velocity observations from two assumed radars that are used in all the experiments. For comparison purpose, the impact of MCEC already contained in the ARPS 3DVAR is also examined.

The DPEC is found in the OSSEs to have small impacts during the first several data assimilation cycles when MCEC is seen to have larger impacts. However, the impact of DPEC becomes more pronounced during the later cycles. Including both DPEC and MCEC in the 3DVAR cost function is found to yield the best analysis of the storm. Sensitivity experiments on the weighting coefficients show that DPEC is not very sensitive to the choice of its weighting coefficient, and the coefficient can be increased or decreased by a factor of 5 without too much effect on the assimilation results. However, large DPEC weight (increase by a factor of 25 in our idealized case) should be avoided because it gives DPEC too much of the total $J$ and evidently degrades data assimilation results.

The new ARPS 3DVAR system with DPEC is further applied to the 5 May 2007 Greensburg tornadic supercell storm. The use of DPEC in this real case is shown to speed up the spinup of convective cells during the intermittent data assimilation process, and improve the ensuing forecast in terms of the general evolution of storm cells and the low-level rotation near the time of observed tornado

Overall, the additional DPEC in the ARPS 3DVAR system has positive impacts on storm-scale 3DVAR data assimilation of Doppler radar data, and on the subsequent forecast. In the future, the system will be tested with more real data cases to hopefully demonstrate the robustness of the conclusions.

Acknowledgments. This work was supported by NSF Grants ATM-0331756, ATM-0738370, NSF ATM0530814, NSF AGS-0802888, and NOAA's Warn-onForecast Project. The third author was also supported by NSF OCI-0905040, AGS-0941491, and AGS-1046171. Robin Tanamachi kindly provided the manually dealiased radial velocity dataset of the Dodge City, Kansas (KDDC) radar. Computations were performed at the Pittsburgh Supercomputing Center (PSC) and Oklahoma Supercomputing Center for Research and Education (OSCER). We appreciate three anonymous reviewers for their very thoughtful comments, which helped significantly improve the quality of this paper from its original form.

\section{APPENDIX}

\section{List of Acronyms and Variables}

3DVAR Three-dimensional variational data assimilation

4DVAR Four-dimensional variational data assimilation

ARPS Advanced Regional Prediction System

CDT Central Daylight Time

DA Data assimilation

DPEC Diagnostic pressure equation constraint

EF Enhanced Fujita scale

EnKF Ensemble Kalman filter

ETS Equitable threat scores

MCEC Mass continuity equation constraint 


$\begin{array}{lll}\text { MM5 } & \text { Fifth-generation Pennsylvania State Uni- } & \mathbf{j} \\ & \text { versity (PSU)-National Center for At- } & \mathbf{k} \\ & \text { mospheric Research (NCAR) Mesoscale } & f \\ \text { NWP } & \text { Model } & \tilde{f} \\ \text { OSSE } & \text { Observational System Simulation Experi- } & \Omega \\ & \text { ment } & \mathrm{O}(\ldots) \\ \text { RHS } & \text { Right-hand side } & \|\ldots\| \\ \text { RMS } & \text { Root-mean square } & V_{r} \\ \text { WSR-88D } & \text { Weather Surveillance Radar-1988 } & \psi \\ & \quad \text { Doppler (radar) } & \\ J & \text { Total cost function } & \\ J_{b} & \text { Background term in the total cost function } & \end{array}$

Unit vector in $y$ direction

Unit vector in $z$ direction

Coriolis coefficient

Coriolis coefficient

Angular velocity of the earth

Latitude

Big O notation

Euclidean norm

Radial velocity

Elevation angle of a radar beam
$J_{o}$

$J_{c}$

$\mathbf{x}$

$\mathbf{x}^{b}$

B

H

$\mathbf{y}^{o}$

$\mathbf{R}$

$\delta \mathrm{x}$

V

$(\ldots)^{\mathrm{T}} \quad$ Transpose operator

$u$

$v$

$w$

$\theta$

$p$

$q_{v}$

$Q$

$P$

$\nabla$

$\mathbf{E}$

$\nabla^{2}$

$(\cdots)^{\prime}$

$\overline{(\cdots)}$

V

$g$

$\partial$

$c_{s}$

$\varepsilon$

$q_{\text {liquid }+ \text { ice }}$

C

D function

Analysis vector

Background vector

Observation vector function)

Vertical velocity

Pressure

MCEC

DPEC

Gradient operator

Laplace operator

Base state

Wind vector water vapor and ice phases
Observation term in the total cost function

Equation constraint term in the total cost

Background error covariance matrices

Observation forward operator

Observation error covariance matrices

Analysis increment vector

Analysis vector (in incremental form cost

$u$ component wind (in $x$ direction)

$v$ component wind (in $y$ direction)

Potential temperature

Water vapor mixing ratio

Dot product operator

Forcing term of the vector Euclidian momentum equations

Deviation from the base state

Gravitational constant

Partial derivative operator

Acoustic wave speed

Ratio of the gas constants for day air and

Mixing ratios for precipitations in liquid

Coriolis force vector

The vector containing subgrid scale turbulence and computational mixing terms

Unit vector in $x$ direction
Aksoy, A., D. C. Dowell, and C. Snyder, 2009: A multicase comparative assessment of the ensemble Kalman filter for assimilation of radar observations. Part I: Storm-scale analyses. Mon. Wea. Rev., 137, 1805-1824.

— _ - and — 2010: A multicase comparative assessment of the ensemble Kalman filter for assimilation of radar observations. Part II: Short-range ensemble forecasts. Mon. Wea. Rev., 138, 1273-1292.

Buehner, M., 2005: Ensemble-derived stationary and flowdependent background-error covariances: Evaluation in a quasi-operational NWP setting. Quart. J. Roy. Meteor. Soc., 131, 1013-1043.

Caya, A., J. Sun, and C. Snyder, 2005: A comparison between the 4D-VAR and the ensemble Kalman filter techniques for radar data assimilation. Mon. Wea. Rev., 133, 3081-3094.

Crook, N. A., 1994: Numerical simulations initialized with radarderived winds. Part I: Simulated data experiments. Mon. Wea. Rev., 122, 1189-1203.

— and J. D. Tuttle, 1994: Numerical simulations initialized with radar-derived winds. Part II: Forecasts of three gust front cases. Mon. Wea. Rev., 122, 1214-1217.

Dawson, D. T., II, M. Xue, J. A. Milbrandt, and M. K. Yau, 2010: Comparison of evaporation and cold pool development between single-moment and multi-moment bulk microphysics schemes in idealized simulations of tornadic thunderstorms. Mon. Wea. Rev., 138, 1152-1171.

Dowell, D. C., L. J. Wicker, and C. Snyder, 2011: Ensemble Kalman filter assimilation of radar observations of the 8 May 2003 Oklahoma City supercell: Influences of reflectivity observations on storm-scale analyses. Mon. Wea. Rev., 139, 272-294.

Gal-Chen, T., 1978: A method for the initialization of the anelastic equations: Implications for matching models with observations. Mon. Wea. Rev., 106, 587-606.

Gao, J.-D., M. Xue, A. Shapiro, and K. K. Droegemeier, 1999: A variational method for the analysis of three-dimensional wind fields from two Doppler radars. Mon. Wea. Rev., 127, 2128-2142.

,,,--- Q. Xu, and K. K. Droegemeier, 2001: Threedimensional simple adjoint velocity retrievals from single Doppler radar. J. Atmos. Oceanic Technol., 18, 26-38.

,,-- K. Brewster, and K. K. Droegemeier, 2004: A threedimensional variational data analysis method with recursive filter for Doppler radars. J. Atmos. Oceanic Technol., 21, 457469.

Hamill, T. M., and C. Snyder, 2000: A hybrid ensemble Kalman filter-3D variational analysis scheme. Mon. Wea. Rev., 128, 2905-2919.

Hane, C. E., and B. C. Scott, 1978: Temperature and pressure perturbations within convective clouds derived from detailed 
air motion information: Preliminary testing. Mon. Wea. Rev., 106, 654-661.

$\mathrm{Hu}$, M., and M. Xue, 2007a: Analysis and prediction of 8 May 2003 Oklahoma City tornadic thunderstorm and embedded tornado using ARPS with assimilation of WSR-88D radar data. Preprints, 22nd Conf. on Weather Analysis and Forecasting/ 18th Conf. on Numerical Weather Prediction, Salt Lake City, UT, Amer. Meteor. Soc., 1B.4. [Available online at http:// ams.confex.com/ams/pdfpapers/123683.pdf.]

— , and — 2007b: Impact of configurations of rapid intermittent assimilation of WSR-88D radar data for the 8 May 2003 Oklahoma City tornadic thunderstorm case. Mon. Wea. Rev., 135, 507-525.

, and K. Brewster, 2006a: 3DVAR and cloud analysis with WSR-88D level-II data for the prediction of Fort Worth tornadic thunderstorms. Part I: Cloud analysis and its impact. Mon. Wea. Rev., 134, 675-698.

- - - J. Gao, and K. Brewster, 2006b: 3DVAR and cloud analysis with WSR-88D level-II data for the prediction of Fort Worth tornadic thunderstorms. Part II: Impact of radial velocity analysis via 3DVAR. Mon. Wea. Rev., 134, 699-721.

Ide, K., P. Courtier, M. Ghil, and A. Lorenc, 1997: Unified notation for data assimilation: Operational, sequential and variational. J. Meteor. Soc. Japan, 75, 181-189.

Li, Y., X. Wang, and M. Xue, 2012: Assimilation of radar radial velocity data with the WRF ensemble-3DVAR hybrid system for the prediction of Hurricane Ike (2008). Mon. Wea. Rev., in press.

Lin, Y.-L., R. D. Farley, and H. D. Orville, 1983: Bulk parameterization of the snow field in a cloud model. J. Climate Appl. Meteor., 22, 1065-1092.

Liou, Y.-C., 2001: The derivation of absolute potential temperature perturbations and pressure gradients from wind measurements in three-dimensional space. J. Atmos. Oceanic Technol., 18, 577-590.

_- and Y.-J. Chang, 2009: A variational multiple-Doppler radar three-dimensional wind synthesis method and its impacts on thermodynamic retrieval. Mon. Wea. Rev., 137, 3992-4010.

— , T.-C. C. Wang, and K.-S. Chung, 2003: A three-dimensional variational approach for deriving the thermodynamic structure using Doppler wind observations-An application to a subtropical squall line. J. Appl. Meteor., 42, 1443-1454.

Liu, H., and M. Xue, 2006: Retrieval of moisture from slant-path water vapor observations of a hypothetical GPS network using a three-dimensional variational scheme with anisotropic background error. Mon. Wea. Rev., 134, 933-949.

,-- R. J. Purser, and D. F. Parrish, 2007: Retrieval of moisture from simulated GPS slant-path water vapor observations using 3DVAR with anisotropic recursive filters. Mon. Wea. Rev., 135, 1506-1521.

Lorenc, A., 2003: The potential of the ensemble Kalman filter for NWP-A comparison with 4D-Var. Quart. J. Roy. Meteor. Soc., 129, 3183-3203.

McCarthy, D., L. Ruthi, and J. Hutton, 2007: The Greensburg, KS tornado. Preprints, 22th Conf. on Weather Analysis and Forecasting and 18th Conf. on Numerical Weather Prediction, Park City, UT, Amer. Meteor. Soc., J2.4. [Available online at http://ams.confex.com/ams/pdfpapers/126927.pdf.]

Protat, A., and I. Zawadzki, 2000: Optimization of dynamic retrievals from a multiple-Doppler radar network. J. Atmos. Oceanic Technol., 17, 753-760.

—_ - and A. Caya, 2001: Kinematic and thermodynamic study of a shallow hailstorm sampled by the McGill bistatic
multiple-Doppler radar network. J. Atmos. Sci., 58, 12221248.

Purser, R. J., W.-S. Wu, D. F. Parrish, and N. M. Roberts, 2003a: Numerical aspects of the application of recursive filters to variational statistical analysis. Part I: Spatially homogeneous and isotropic Gaussian covariances. Mon. Wea. Rev., 131, 1524-1535.

,,,--- and $-2003 \mathrm{~b}$ : Numerical aspects of the application of recursive filters to variational statistical analysis. Part II: Spatially inhomogeneous and anisotropic general covariances. Mon. Wea. Rev., 131, 1536-1548.

Ray, P. S., and Coauthors, 1981: The morphology of severe tornadic storms on 20 May 1977. J. Atmos. Sci., 38, 1643-1663.

Snook, N., M. Xue, and Y. Jung, 2011: Analysis of a tornadic mesoscale convective vortex based on ensemble Kalman filter assimilation of CASA X-band and WSR-88D radar data. Mon. Weat. Rev., 139, 3446-3468.

Snyder, C., and F. Zhang, 2003: Assimilation of simulated Doppler radar observations with an ensemble Kalman filter. Mon. Wea. Rev., 131, 1663-1677.

Stensrud, D. J., and J. Gao, 2010: Importance of horizontally inhomogeneous environmental initial conditions to ensemble storm-scale radar data assimilation and very short-range forecasts. Mon. Wea. Rev., 138, 1250-1272.

Sun, J., 2005: Initialization and numerical forecasting of a supercell storm observed during STEPS. Mon. Wea. Rev., 133, 793-813.

— and N. A. Crook, 1997: Dynamical and microphysical retrieval from Doppler radar observations using a cloud model and its adjoint. Part I: Model development and simulated data experiments. J. Atmos. Sci., 54, 1642-1661.

— and - 1998: Dynamical and microphysical retrieval from Doppler radar observations using a cloud model and its adjoint. Part II: Retrieval experiments of an observed Florida convective storm. J. Atmos. Sci., 55, 835-852.

_, and _ 2001: Real-time low-level wind and temperature analysis using single WSR-88D data. Wea. Forecasting, 16, 117-132.

, D. W. Flicker, and D. K. Lilly, 1991: Recovery of three-dimensional wind and temperature fields from single-Doppler radar data. J. Atmos. Sci., 48, 876-890.

Tong, M., and M. Xue, 2005: Ensemble Kalman filter assimilation of Doppler radar data with a compressible nonhydrostatic model: OSS experiments. Mon. Wea. Rev., 133, 1789-1807.

Wang, X., D. M. Barker, C. Snyder, and T. M. Hamill, 2008a: A hybrid ETKF-3DVAR data assimilation scheme for the WRF model. Part I: Observing system simulation experiment. Mon. Wea. Rev., 136, 5116-5131.

$\longrightarrow,-,-$, and $\longrightarrow, 2008$ b: A hybrid ETKF-3DVAR data assimilation scheme for the WRF model. Part II: Real observation experiment. Mon. Wea. Rev., 136, 5132-5147.

Wang, Z., 1993: Variational data assimilation with 2-D shallow water equations and 3-D FSU global spectral models. Dissertation, Department of Mathematics, Florida State University, $224 \mathrm{pp}$.

Weygandt, S. S., A. Shapiro, and K. K. Droegemeier, 2002a: Retrieval of model initial fields from single-Doppler observations of a supercell thunderstorm. Part I: Single-Doppler velocity retrieval. Mon. Wea. Rev., 130, 433-453.

,$- \ldots$, and $-2002 \mathrm{~b}$ : Retrieval of model initial fields from single-Doppler observations of a supercell thunderstorm. Part II: Thermodynamic retrieval and numerical prediction. Mon. Wea. Rev., 130, 454-476. 
Xiao, Q., Y.-H. Kuo, J. Sun, W.-C. Lee, E. Lim, Y.-R. Guo, and D. M. Barker, 2005: Assimilation of Doppler radar observations with a regional 3DVAR system: Impact of Doppler velocities on forecasts of a heavy rainfall case. J. Appl. Meteor., 44, 768-788.

Xu, Q., H. Gu, and S. Yang, 2001: Simple adjoint method for threedimensional wind retrievals from single-Doppler radar. Quart. J. Roy. Meteor. Soc., 127, 1053-1068.

Xue, M., K. K. Droegemeier, and V. Wong, 2000: The Advanced Regional Prediction System (ARPS)-A multiscale nonhydrostatic atmospheric simulation and prediction tool. Part I: Model dynamics and verification. Meteor. Atmos. Phys., 75, 161-193.

, and Coauthors, 2001: The Advanced Regional Prediction System (ARPS) - A multi-scale nonhydrostatic atmospheric simulation and prediction tool. Part II: Model physics and applications. Meteor. Atmos. Phys., 76, 143-166.

—, D.-H. Wang, J.-D. Gao, K. Brewster, and K. K. Droegemeier, 2003: The Advanced Regional Prediction System (ARPS), storm-scale numerical weather prediction and data assimilation. Meteor. Atmos. Phys., 82, 139-170.

_- M. Tong, and K. K. Droegemeier, 2006: An OSSE framework based on the ensemble square-root Kalman filter for evaluating impact of data from radar networks on thunderstorm analysis and forecast. J. Atmos. Oceanic Technol., 23, 46-66. - and Coauthors, 2008: CAPS realtime storm-scale ensemble and high-resolution forecasts as part of the NOAA Hazardous
Weather Testbed 2008 Spring Experiment. Preprints, 24th Conf. Several Local Storms, Savannah, GA, Amer. Meteor. Soc., 12.12. [Available online at http://ams.confex.com/ams/ pdfpapers/142036.pdf.]

_ , and Coauthors, 2011: Realtime convection-permitting ensemble and convection-resolving deterministic forecasts of CAPS for the hazardous weather testbed 2010 spring experiment. Preprints, 24th Conf. Weather Forecasting/20th Conf. Numerical Weather Prediction, Seattle, WA, Amer. Meteor. Soc., 9A.2. [Available online at http://ams.confex.com/ams/91Annual/webprogram/ Manuscript/Paper183227/Xue_CAPS_2011_SpringExperiment_ 24thWAF20thNWP_ExtendedAbstract.pdf.]

Zhang, F., C. Snyder, and J. Sun, 2004: Impacts of initial estimate and observations on the convective-scale data assimilation with an ensemble Kalman filter. Mon. Wea. Rev., 132, 1238 1253.

— , Y. Weng, J. A. Sippel, Z. Meng, and C. H. Bishop, 2009: Cloud-resolving hurricane initialization and prediction through assimilation of Doppler radar observations with an ensemble Kalman filter. Mon. Wea. Rev., 137, 2105-2125.

Zhao, Q., J. Cook, Q. Xu, and P. R. Harasti, 2006: Using radar wind observations to improve mesoscale numerical weather prediction. Wea. Forecasting, 21, 502-522.

$-, \ldots, \ldots$, and — 2008: Improving short-term storm predictions by assimilating both radar radial-wind and reflectivity observations. Wea. Forecasting, 23, 373-391. 